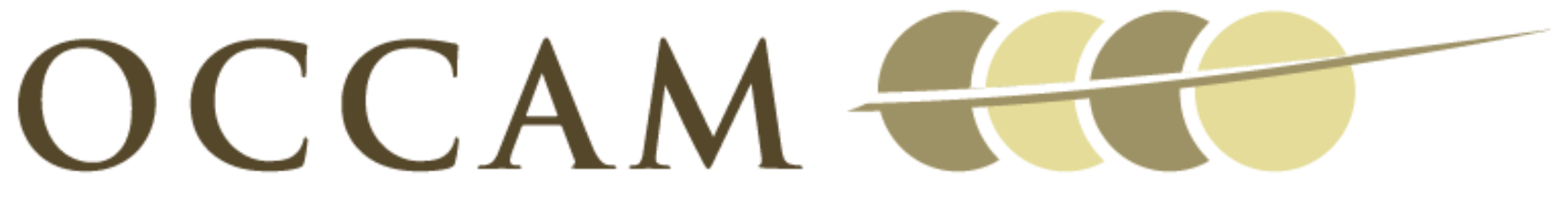

OXFORD CENTRE FOR COLLABORATIVE APPLIED MATHEMATICS

Report Number 10/18

A Mathematical Model of Tumor-Immune Interactions

by

Mark Robertson-Tessi, Ardith El-Kareh, Alain Goriely

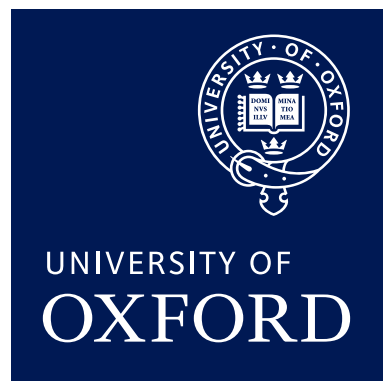

Oxford Centre for Collaborative Applied Mathematics Mathematical Institute 24 - 29 St Giles'

Oxford

OX1 3LB

England 



\title{
A Mathematical Model of Tumor-Immune Interactions
}

\author{
Mark Robertson-Tessi ${ }^{\mathrm{a}}$, Ardith El-Kareh $^{\mathrm{b}}$, Alain Goriely ${ }^{\mathrm{c}}$ \\ ${ }^{a}$ Program in Applied Mathematics, University of Arizona, Tucson, AZ 85721 \\ ${ }^{b} A R L$-Microcirculation Division, University of Arizona, Tucson, AZ 85724 \\ ${ }^{c}$ Oxford Center for Collaborative Applied Mathematics, Mathematical Institute, Oxford, \\ OX2 6HA, UK
}

\begin{abstract}
A mathematical model of the interactions between a growing tumor and the immune system is presented. The equations and parameters of the model are based on experimental and clinical results from published studies. The model includes the primary cell populations involved in effector T-cell mediated tumor killing: regulatory $\mathrm{T}$ cells, helper $\mathrm{T}$ cells, and dendritic cells. A key feature is the inclusion of multiple mechanisms of immunosuppression through the main cytokines and growth factors mediating the interactions between the cell populations. Decreased access of effector cells to the tumor interior with increasing tumor size is accounted for. The model is applied to tumors with different growth rates and antigenicities to gauge the relative importance of various immunosuppressive mechanisms. The most important factors leading to tumor escape are TGF- $\beta$-induced immunosuppression, conversion of helper $\mathrm{T}$ cells into regulatory $\mathrm{T}$ cells, and the limitation of immune cell access to the full tumor at large tumor sizes. The results suggest that for a given tumor growth rate, there is an optimal antigenicity maximizing the response of the immune system. Further increases in antigenicity result in increased immunosuppression, and therefore a decrease in tumor killing rate.
\end{abstract}

Keywords: immunosuppression, regulatory t cells, TGF- $\beta$, IL-10, tumor growth

\section{Introduction and Motivation}

Understanding the interplay between a growing tumor and the host's immune system is fundamental for optimizing current treatments and propos- 
ing new ones. Despite much research into these tumor-immune interactions (Dunn et al., 2002; Villunger and Strasser, 1999), there are many open questions in the field and quantitative theories are relatively few. Although the immune system is known to play a role in the prevention and removal of some tumors, it appears to be incapable of fighting others (Vaage, 1971). Recent research suggests that immunosuppression is a significant obstacle that the immune system faces when fighting a tumor (Chouaib et al., 1997). Since there are many forms of immunosuppression acting at different levels of the immune system, it is important to quantify the effect of each suppressive element at each stage of tumor growth.

Mathematical modeling has been used in conjunction with experimental and clinical results to explore the significance of tumor-immune interactions. Previous work has included models of T cells with interleukin-2 (IL-2) (Kirschner and Panetta, 1998), transforming growth factor beta (TGF- $\beta$ ) (Arciero et al., 2004), regulatory T cells (Tregs) (Leon et al., 2007), and natural killer cells (de Pillis et al., 2005).

The mathematical model presented in this paper examines the T-cell response to a tumor. Several new features are included in the model. The effects of multiple suppressive elements are examined, so that their respective effect can be quantified at different stages of tumor growth. The growth law of the tumor is modeled as a hybrid between exponential and power law growth. Immune cell access to the interior of a large tumor is limited, based on perfusion data. Helper $\mathrm{T}$ cells are also included in the model, since they affect the dynamics of the immune response. In addition, helper $\mathrm{T}$ cells can be converted into regulatory $\mathrm{T}$ cells by TGF- $\beta$. Finally, all the parameters were estimated from experimental and clinical data. Although these parameters necessarily come from different systems, using realistic parameters ensures that the model operates within a biologically reasonable regime.

The paper is organized as follows: Section 2 briefly outlines the biological processes involved in mounting a $\mathrm{T}$ cell immune response to a tumor. The mathematical model is presented in Section 3, and the analysis of the model is described in Section 4. Conclusions and ideas for further development of this model are given in Section 5 .

\section{Tumor Immune Interactions}

Many murine and in vitro studies have shown that tumor cells can be killed by immune system cells (Hellstrom and Hellstrom, 1974). It is plau- 
sible that in humans, some tumors which begin to grow in the body are removed by the immune system without external intervention. Of course, these tumors are not observed, since they are removed before they are of detectable size. In murine studies, immunodeficient mice have been shown to have increased incidence of cancers (Dunn et al., 2006), and human patients taking immunosuppressive agents also show increased susceptibility to many cancer types (Wimmer et al., 2007), suggesting that the immune system plays a role in the spontaneous removal of some small tumors.

Although other immune cells play a role in fighting cancer, $\mathrm{T}$ cells have been suggested as one of the principal methods that the body uses to combat a tumor (Hellstrbm and Hellstrom, 1991; Rosenberg et al., 1986). A diagram of the interactions between the cancer and the T-cell immune system is shown in Figure 1. As the tumor grows, it provokes an immune response from the $\mathrm{T}$ cells in the host. The strength of this response depends on the tumor's antigenicity, which describes how much antigen a tumor presents, and also how sensitive the immune system is to this antigen.

In the cases where a tumor reaches a clinical size on the order of $10^{9}$ to $10^{11}$ cells, there may be several mechanisms by which the tumor may have escaped the immune response. One possibility is that the antigens presented by the tumor are not strong or prevalent enough to elicit an immune response. In this case, the tumor is not recognized as a significant threat by the immune system. Another possibility is that antigen may be present in sufficient strength, but the immunosuppressive elements may be reducing the efficacy of the immune system. Research has shown that some murine tumors which have grown without check can be removed by the immune system, after certain suppressive factors are blocked (Gorelik and Flavell, 2001; Viehl et al., 2006). This suggests that the immune system has the capability to fight the tumor, but that the suppressive effects prevent proper action of the $\mathrm{T}$ cells on the tumor.

In a simplified model of an immune response to a tumor, excluding any immunosuppression, the system can be seen as a predator-prey system. The T-cell response would fall into three categories. If the antigenicity is high enough, the tumor will provoke a large immune response, and the $\mathrm{T}$ cells will fully remove the tumor. If the antigenicity is very low, then the T-cell reaction will be minimal. The immune system may slow down the tumor growth rate, but the tumor will continue to grow without bound. At a moderate antigenicity, there may exist an equilibrium state, where the tumor growth rate and the immune system killing rate balance each other. 
The addition of immunosuppression adds a complex negative feedback loop to the activation of the immune system. The tumor provokes an immune response as described above, but also promotes suppression of the immune system, which will diminish the T-cell response. There are a number of mechanisms for this suppression; the literature suggests that the significant sources of T-cell immunosuppression are Tregs (Viehl et al., 2006; Frumento et al., 2006), TGF- $\beta$ (Gorelik and Flavell, 2001; Kobie and Akporiaye, 2003; Wojtowicz-Praga, 2003), and interleukin-10 (IL-10) (Avradopoulos et al., 1997; Hsieh et al., 2000). These immunosuppressive elements act in different ways on the system, and have different levels of activity at different stages of tumor growth. The model of these interactions is presented in the following section.

\section{A Mathematical Model}

\subsection{Variables}

Since the immune system and its interactions with tumor cells are highly complicated, a full model which includes all the significant cell types and signaling molecules is unrealistic. However, if the model is too simple, the results will be trivial and will likely lack the complex dynamics observed in experimental and clinical settings.

In selecting the variables to consider in this model, several principles were used as a guide. First, the values of the parameters appearing in an equation must be available from experimental data which allowed for a reasonable estimation of a biologically appropriate range. Second, enough cell types and interactions must be included to capture the short time scale dynamics of T-cell proliferation observed in experiments. The activation and proliferation of T-cells is subject to competition between the different T-cell types, and involves several phases which have different characteristic time scales. Third, the immune system must respond naturally in the absence of tumorinduced suppressive terms, as opposed to only acting reasonably when these suppressive effects are applied.

The model presented here uses 12 biological variables: 9 cell types, and 3 cytokines. Naturally, tumor cells are included, and are denoted by the variable $T(t)$, where $t$ stands for time in days. The heterogeneity of the interactions between tumor cells and the immune system is an important effect that is neglected in this model due to a lack of specific experimental data. While there is no explicit spatial component to the model at this time, 
we do account for tumor vasculature by its action on tumor growth rate and immune system access. The model assumes that the tumor is perfused from the outside. Therefore, immune system cells will only reach a certain layer of the tumor once the tumor becomes large compared to the perfusion depth.

In order to investigate the effects of the T-cell response, the model must include $\mathrm{CD}^{+}$effector $\mathrm{T}$ cells, which directly kill the tumor cells. In addition, $\mathrm{CD}^{+}{ }^{+} \mathrm{CD} 25^{+}$Foxp3 $^{+}$regulatory $\mathrm{T}$ cells are included in order to account for immunosuppression, since they produce suppressive cytokines and also act directly to reduce effector T-cell killing rates.

Stimulation of $\mathrm{T}$ cells occurs in the lymph nodes, and begins when antigenpresenting cells (APCs) bearing tumor antigens interact with the T cells. In this model, dendritic cells are the chosen APCs, since they are the primary mechanism for T-cell activation (Roitt and Delves, 2001).

A third population of $\mathrm{T}$ cells, $\mathrm{CD} 4^{+}$helper $\mathrm{T}$ cells, is included to assist with cytokine production and dendritic cell licensing. The inclusion of helper $\mathrm{T}$ cells as a distinct population is necessary for two reasons. First, the helper cells license the dendritic cells, which prevents an unrealistically rapid development of effector $\mathrm{T}$ cells. Second, the helper cells are converted to Tregs by TGF- $\beta$, which is a key immunosuppressive mechanism observed in experiments (Liu et al., 2007b).

All three types of $\mathrm{T}$ cells are all specific to the tumor antigen, and are a small fraction of the total T-cell repertoire within a host. Each of the three T-cell populations are broken into three subpopulations. The memory T cell population $\left(M_{E}, M_{H}\right.$, and $M_{R}$, which are all assumed to be constant) is a pool of T-cell precursors, which are activated by dendritic cells. There is a short lived activation phase $\left(A_{E}(t), A_{H}(t)\right.$, and $\left.A_{R}(t)\right)$ where T-cell proliferation occurs. The fully functional T-cell phase $(E(t), H(t)$, and $R(t))$ consists of the cells that kill the tumor cells, produce cytokines, and suppress the immune response. These subpopulations capture the different time scales of the cellular phases, and contribute three constants and six variables to the model.

The dendritic cell population has two subpopulations. The unlicensed state, $U(t)$, is a mature dendritic cell that interacts with helper cells. After licensing, the dendritic cell, $D(t)$, is free to interact with all T cells, causing them to activate.

The T-cell and dendritic cell populations are subdivided in order to account for the dynamic delays in activation observed in experiments (Mempel et al., 2004). In addition, the subdivision allows the various immunosuppres- 
sive effects which occur at different steps in the T-cell activation sequence to be properly included.

The selection of relevant cytokines was motivated by the literature. IL-2, denoted by the variable $C(t)$, is not only a key proliferative cytokine for T cells, but is also a current option for immunotherapy (Dudley et al., 2002; Rosenberg and Lotze, 1986). It is necessary to include IL-2 in order to include other suppressive effects, as IL-2 plays a role in balancing the ratio of Tregs to other T cells (Setoguchi et al., 2005). For the suppressive cytokines, TGF- $\beta$, denoted by $S(t)$, and IL-10, denoted by $I(t)$, are considered to be important suppressors of T-cell activity, as described above, and are produced by both tumor cells (Hsieh et al., 2000) and Tregs (Jarnicki et al., 2006).

The subset of equations describing the response of the immune system to antigen functions realistically in the absence of tumor-induced immunosuppression. This ensures that any tumor-associated suppressive effects are properly scaled with respect to the innate immune response. The tumorimmune interactions and the associated suppression caused by Tregs, TGF- $\beta$, and IL-10 are added to the base model of T-cell activation.

While other suppressive cytokines and cell types exist, these 12 populations are the important players in the evolution of the tumor-immune system and have been described extensively in experimental and clinical literature.

Figure 2 shows a detailed view of the interactions modeled in this paper. The tumor cells produce antigen, which is collected by dendritic cells. These dendritic cells then interact with three T-cell types: effector $\mathrm{T}$ cells, helper $\mathrm{T}$ cells, and Tregs. The Tregs produce suppressive molecules TGF- $\beta$ and IL-10 and also directly suppress the activity of the immune system (Piccirillo et al., 2002). All three of these T-cell types are expanded due to antigen presentation by the dendritic cells. Tregs have an additional expansion mechanism. Research has shown that TGF- $\beta$ can promote the conversion of helper T cells into Tregs (Liu et al., 2007b), which adds another feedback mechanism to the model.

\subsection{Equations}

\subsubsection{Tumor Cells}

In our model, the evolution of tumor cells $(T)$ is governed by

$$
\dot{T}=\frac{T}{\left(\left(\frac{1}{\gamma_{1}}\right)^{p}+\left(\frac{T^{1-m}}{\gamma}\right)^{p}\right)^{1 / p}}-\frac{r_{0} T^{*}}{\left(1+k_{2} \frac{T^{*}}{E}\right)} \cdot \frac{1}{\left(1+k_{3} \frac{R}{E}\right)\left(1+\frac{S}{S_{1}}\right)},
$$


where $\gamma_{1}$ is given by

$$
\gamma_{1}=\gamma\left(T_{1}\right)^{m-1}
$$

and $T^{*}$ is given by

$$
T^{*}=\frac{T}{\left(1+\left(\frac{T^{1-n}}{k_{1}}\right)^{p}\right)^{1 / p}} .
$$

The first term on the right hand side of Eq. (1) accounts for tumor growth. Previously, the growth law for a tumor has been modeled in several ways, including logistic (Arciero et al., 2004; de Pillis et al., 2005; Kuznetsov et al., 1994) and Gompertzian (Norton, 1988) models. Experimental data for different types of tumors under different conditions exhibit different growth curves, so there is no universal choice in general. Power law growth has been shown to be a good fit for breast cancer (Hart et al., 1998). In this paper, the growth is modeled in two phases. When the tumor is small, the growth is exponential with coefficient $\gamma_{1}$; when the tumor is large, the growth follows a power law with exponent $m$ and coefficient $\gamma$. The transition between these two modes of growth is smooth. The parameters $\gamma$ and $\gamma_{1}$ are chosen so that the growth rate is the same at the transition, chosen to be $T_{1}=10^{6}$ cells (Steele, 1977). The exponent $p$ determines smoothness of the transition between the two growth laws, and does not qualitatively affect the results. The parameter $\gamma$ will be used as one of the two fundamental control parameters in the model, in order to account for tumors growing at different rates. There is no explicit term for natural death of tumor cells; rather, it is taken into account in the growth term. Although there is evidence that TGF- $\beta$ can promote tumor growth (Fitzpatrick et al., 1996), this effect is not included in this study.

The second term of Eq. (1) describes tumor cell kill by effector cells $(E)$, which is the only mechanism for tumor cell death in the model. The first fraction in this term accounts for tumor cell death in the absence of enhancing or suppressing effects. The parameter $r_{0}$ is the rate at which an effector cell kills a tumor cell. The total rate of tumor cell killing depends on the ratio of tumor cells and effector cells. When this ratio is high (effector cell numbers are small compared to tumor cell numbers), each effector cell has access to many tumor cells, and thus the killing rate is on the order of $\frac{r_{0} E}{k_{2}}$, proportional to the number of effector cells. When effector cell counts are much larger than tumor cell counts, the killing rate saturates to a level dependent on tumor cells only $\left(r_{0} T^{*}\right)$, since additional effector cells entering the system will not 
contribute significantly to the tumor cell killing rate. The parameter $k_{2}$ controls the shape of the interaction when cell counts are comparable in number.

The variable $T^{*}$ represents the number of tumor cells which are accessible by the immune system, and combines different effects. Poor vascularization of a growing tumor limits the access of immune cells to the entire tumor (Jain and Ward-Hartley, 1984; Tannock et al., 2002). For small tumor sizes, the entire tumor is accessible to the immune cells. As the tumor grows larger, only an outer shell of the tumor is sufficiently perfused with blood vessels to allow immune cell access (Jain and Ward-Hartley, 1984). This transition occurs at approximately the same size as the transition in the growth law, since both have a dependence on blood vessel perfusion depths. In the model, $T^{*}$ is used in place of $T$ whenever the interactions are dependent on the spatial access to the tumor cells. For example, production of TGF- $\beta$ can be expected of all functional tumor cells, and therefore $T$ is used for that process. The interaction of $\mathrm{CD}^{+}$effector $\mathrm{T}$ cells and tumor cells, however, requires physical contact, and therefore effector cells primarily encounter the perfused tumor mass, $T^{*}$.

The second fraction in the second term of Eq. (1) represents the suppressive effects of Tregs and TGF- $\beta$ on the cytotoxicity. The term $1+k_{3} \frac{R}{E}$ represents the effect of Tregs $(R)$ on the effector cells' killing rate. The suppression is ratio dependent, with parameter $k_{3}$ controlling the shape of the interaction, so that increased Treg to effector $\mathrm{T}$ cell ratios will decrease the tumor cell killing rate. The term $1+\frac{S}{S_{1}}$ represents suppression of effector cell activity due to TGF- $\beta(S)$, which has been shown to reduce the cytotoxicity of effector T cells (Thomas and Massagué, 2005). The parameter $S_{1}$ is the TGF- $\beta$ concentration where killing is suppressed by half.

\subsubsection{Dendritic Cells}

Mature dendritic cells evolve according to

$$
\dot{U}=\frac{a T^{*}}{\left(1+\frac{I}{I_{1}}\right)\left(1+\frac{R}{R_{1}}\right)}-\frac{\lambda U}{1+\frac{U}{M_{H}}}-\delta_{U} U
$$

and

$$
\dot{D}=\frac{\lambda U}{1+\frac{U}{M_{H}}}-\delta_{D} D
$$


where $U$ represents the number of unlicensed dendritic cells, and $D$ is the number of licensed dendritic cells. The first term of Eq. (4) describes the maturation of dendritic cells by encounter with tumor antigen. The antigenicity of a tumor, denoted by the parameter $a$, is known to vary highly among different types of tumors (Chen et al., 1994; Kripke, 1974), and is taken as the second control parameter in this study.

The maturation process is inhibited by Tregs and IL-10 (Larmonier et al., 2007), represented by the two suppressive denominator terms. Following our previous discussion of effector cells, the difficulty of accessing the tumor interior by immune cells is reflected by the use of $T^{*}$. The model assumes that there is a constant pool of immature dendritic cells which circulate in the body searching for antigens. This constant is effectively included in the parameter $a$.

The second term of Eq. (4) and first term of Eq. (5) account for the licensing of dendritic cells upon encounter with helper $\mathrm{T}$ cells. This licensing process depends on the ratio of the two cell types. For small numbers of dendritic cells, licensing helper $\mathrm{T}$ cells are abundant, and the process goes as $\lambda U$. For high numbers of dendritic cells, the rate is limited by the availability of helper cells, so the licensing is on the order of $\lambda M_{H}$. Eqs. (4) and (5) both include terms to account for cell death or deactivation after a characteristic time period, given by $\delta_{U}$ and $\delta_{D}$.

\subsubsection{Memory T cells}

All three types of $\mathrm{T}$ cells in the model are activated in the same basic way. The memory cells enter a brief activation phase where proliferation is rapid, and then they become fully functional $\mathrm{T}$ cells. The total memory population $M$ is defined by

$$
M=M_{E}+M_{H}+M_{R}
$$

where the right hand terms are the constant populations of effector memory $\mathrm{T}$ cells, helper memory $\mathrm{T}$ cells, and Treg memory cells, respectively.

\subsection{4. $C D 8^{+}$Effector $T$ cells}

$\mathrm{CD}^{+}$effector $\mathrm{T}$ cells follow

$$
\dot{A_{E}}=\frac{\alpha_{1} M_{E}}{1+k_{4} \frac{M}{D}}-\delta_{A} A_{E}
$$


and

$$
\dot{E}=\frac{\alpha_{2} A_{E} C}{\left(1+\frac{S}{S_{2}}\right)\left(C_{1}+C\right)}-\delta_{E} E .
$$

The first term of Eq. (7) activates the effector memory cells, in the presence of mature licensed dendritic cells. Again, this process is ratio dependent. As

dendritic cell numbers increase, the term saturates to $\alpha_{1} M_{E}$. The second term describes the expiration of the activated phase of these cells.

The first term of Eq. (8) gives the proliferation of CD8+ effector cells. This depends on the presence of activated cells and the proliferative cytokine IL-2 $(C)$. As IL-2 increases, the level of proliferation reaches a maximum of $\alpha_{2} A_{E}$ in the absence of suppression. TGF- $\beta(S)$ suppresses T-cell proliferation (Thomas and Massagué, 2005; McKarns and Schwartz, 2005), so we include the parameter $S_{2}$ to account for this effect. The second term accounts for effector cell death or inactivation, with parameter $\delta_{E}$.

\subsection{5. $C D 4^{+}$Helper $T$ cells}

Helper $\mathrm{T}$ cells follow a similar activation path as effector $\mathrm{T}$ cells:

$$
\dot{A_{H}}=\frac{\alpha_{3} M_{H}}{1+k_{4} \frac{M}{(U+D)}}-\delta_{A} A_{H}
$$

and

$$
\dot{H}=\frac{\alpha_{4} A_{H} C}{\left(1+\frac{S}{S_{2}}\right)\left(C_{1}+C\right)}-\frac{\alpha_{7} H S}{S_{3}+S}-\delta_{H} H .
$$

These two equations differ from those for the effector $\mathrm{T}$ cells in two ways. First, the activation of memory helper cells can be prompted by both licensed and unlicensed dendritic cells (Smith et al., 2004). Second, helper T cells can be converted into Tregs in the presence of TGF- $\beta$, and this is represented by the second term in Eq. (10). As TGF- $\beta$ increases, the rate of change from helper cells to Tregs saturates at $\alpha_{7} H$.

\subsection{6. $\mathrm{CD}^{+} \mathrm{CD} 25^{+} \mathrm{Foxp}^{+}$Regulatory T cells}

Tregs follow a similar pair of equations:

$$
\dot{A_{R}}=\frac{\alpha_{5} M_{R}}{1+k_{4} \frac{M}{D}}-\delta_{A} A_{R}
$$


and

$$
\dot{R}=\frac{\alpha_{6} A_{R} C}{\left(C_{1}+C\right)}+\frac{\alpha_{7} H S}{S_{3}+S}-\delta_{R} R .
$$

The principal difference here is that Treg proliferation is not suppressed by TGF- $\beta$, in contrast to the other T-cell populations.

\subsubsection{Cytokines and Molecules}

The proliferative cytokine IL-2 is produced by activated helper cells, and the concentration is given by

$$
\dot{C}=\frac{p_{C} A_{H}}{\left(1+\frac{S}{S_{4}}\right)\left(1+\frac{I}{I_{2}}\right)}-\frac{C}{\tau_{C}} .
$$

The first term of Eq. (13) represents the production of IL-2 by the activated helper cells. This production is inhibited by TGF- $\beta$ (Thomas and Massagué, 2005; McKarns and Schwartz, 2005). The second term represents the removal of IL-2 from the system, with characteristic time $\tau_{C}$.

TGF- $\beta$ and IL-10 are produced by both tumor cells (Alleva et al., 1994) and Tregs (Jarnicki et al., 2006), and follow

$$
\dot{S}=p_{1} R+p_{2} T-\frac{S}{\tau_{S}},
$$

and

$$
\dot{I}=p_{3} R+p_{4} T-\frac{I}{\tau_{I}} .
$$

The first terms of each equation represent the production by Tregs, the second terms represent production by tumor cells, and the final terms represent the removal from the system with characteristic times $\tau_{S}$ and $\tau_{I}$.

For convenience, all 12 equations are collected here.

$$
\begin{aligned}
& \dot{T}=\frac{T}{\left(\left(\frac{1}{\gamma_{1}}\right)^{p}+\left(\frac{T^{1-m}}{\gamma}\right)^{p}\right)^{1 / p}}-\frac{r_{0} T^{*}}{\left(1+k_{2} \frac{T^{*}}{E}\right)} \cdot \frac{1}{\left(1+k_{3} \frac{R}{E}\right)\left(1+\frac{S}{S_{1}}\right)} \\
& \dot{U}=\frac{a T^{*}}{\left(1+\frac{I}{I_{1}}\right)\left(1+\frac{R}{R_{1}}\right)}-\frac{\lambda U}{1+\frac{U}{M_{H}}}-\delta_{U} U
\end{aligned}
$$




$$
\begin{aligned}
& \dot{D}=\frac{\lambda U}{1+\frac{U}{M_{H}}}-\delta_{D} D \\
& \dot{A_{E}}=\frac{\alpha_{1} M_{E}}{1+k_{4} \frac{M}{D}}-\delta_{A} A_{E} \\
& \dot{E}=\frac{\alpha_{2} A_{E} C}{\left(1+\frac{S}{S_{2}}\right)\left(C_{1}+C\right)}-\delta_{E} E \\
& \dot{A_{H}}=\frac{\alpha_{3} M_{H}}{1+k_{4} \frac{M}{(U+D)}}-\delta_{A} A_{H}, \\
& \dot{H}=\frac{\alpha_{4} A_{H} C}{\left(1+\frac{S}{S_{2}}\right)\left(C_{1}+C\right)}-\frac{\alpha_{7} H S}{S_{3}+S}-\delta_{H} H \\
& \dot{A_{R}}=\frac{\alpha_{5} M_{R}}{1+k_{4} \frac{M}{D}}-\delta_{A} A_{R} \\
& \dot{R}=\frac{\alpha_{6} A_{R} C}{\left(C_{1}+C\right)}+\frac{\alpha_{7} H S}{S_{3}+S}-\delta_{R} R . \\
& \dot{C}=\frac{p_{C} A_{H}}{\left(1+\frac{S}{S_{4}}\right)\left(1+\frac{I}{I_{2}}\right)}-\frac{C}{\tau_{C}} . \\
& \dot{S}=p_{1} R+p_{2} T-\frac{S}{\tau_{S}}, \\
& \dot{I}=p_{3} R+p_{4} T-\frac{I}{\tau_{I}},
\end{aligned}
$$


where

$\gamma_{1}=\gamma T_{1}^{m-1}$

and

$T^{*}=\frac{T}{\left(1+\left(\frac{T^{1-n}}{k_{1}}\right)^{p}\right)^{1 / p}}$.

This is a system of 12 ODEs with 41 parameters. The model has two control parameters $\gamma$ and $a$ representing, respectively, growth rate and antigenicity. The remaining 39 parameters are given in Tables 1 through 4, following the analysis given in the next section.

\subsection{Parameter Estimation}

The parameter $m$ was taken directly from Hart et al. (Hart et al., 1998). Using this power law exponent, $\gamma$ was estimated, using measurements of tumor doubling times from various studies (Friberg and Mattson, 1997; Iwashita et al., 1998; Jackson et al., 1984; Peer et al., 1993). Since the doubling time for a power law depends on the size of the tumor, we assumed that the clinical tumors were of typical detectable size when the doubling times were measured (approximately $1 \mathrm{~cm}$ in diameter, or $10^{9}$ cells). Growth rates are highly variable, even among patients with the same type of tumor, so we provide a range of reasonable values in Table 1 , based on these studies. The parameter $\gamma_{1}$ was calculated from the value of $\gamma$ using Eq. 28 so that the growth rates matched at the transition size $T_{1}$.

The parameters $r_{0}$ and $k_{2}$ were taken from T-cell lysis data presented in (Dudley et al., 2002). The level of tumor cell lysis at high T-cell to tumor cell ratios provided $r_{0}$, and the slope at lower ratios allows for the calculation of $k_{2}$. The suppression of tumor cell killing by TGF- $\beta$ was calculated from data (Thomas and Massagué, 2005) that measured the effectiveness of tumor lysis in the presence and absence of TGF- $\beta$. The parameter $S_{1}$ represents the concentration at which lytic capability is cut by half.

The value of the parameter $a$, which represents the antigenicity of a tumor, exhibits significant variation between different tumor types (Kripke, 1974). To account for the large intertumor heterogeneity in both growth rate and antigenicity, ranges of values are used for these parameters. 
The suppression of dendritic cell maturation by IL-10 and Treg cells was calculated from experiments in Larmonier et al. (Larmonier et al., 2007). Expression of the maturation marker Interleukin-12 (IL-12) was measured in dendritic cell cultures. The presence of IL-10, TGF- $\beta$ and Tregs all partially abrogated the maturation process. The data for TGF- $\beta$ were not sufficient to establish a suppressive term in Eq. 17, but parameters $I_{1}$ and $R_{1}$ for IL10 and Tregs were calculated. Data from Smith et al. (Smith et al., 2004) provided the parameter $\lambda$. The death rate of mature dendritic cells, $\delta_{U}$, was taken from experiments measuring the turnover of dendritic cells that had been primed with antigen (Liu et al., 2007a). The parameter $\delta_{D}$, which represents the expiration of antigen presentation of the licensed dendritic cells, was calculated using data from Mempel et al. (Mempel et al., 2004).

The parameters relating to $\mathrm{CD}^{+}$and $\mathrm{CD}^{+}$T-cell activation and proliferation were calculated by separating the equations relating to these populations and finding parameters which resulted in the proper dynamics as outlined by Mempel et al. (2004). The typical ratio of $\mathrm{CD}^{+}$to $\mathrm{CD}^{+} \mathrm{T}$ cells in a healthy individual is approximately 2:1 (Taylor et al., 1989); this ratio is maintained with the choice of activation parameters. The death rates of the $\mathrm{T}$ cells were chosen so that proper numbers of cells were activated upon antigen presentation (Yates and Callard, 2001).

The parameter $k_{4}$ relates to the number of $\mathrm{T}$ cells that can interact with a dendritic cell at any given time. This value can vary, and here it is assumed to be approximately 5 (De Boer and Perelson, 1994); $k_{4}$ therefore controls the activation of $\mathrm{T}$ cells when dendritic cell numbers are comparable to $\mathrm{T}$ cell numbers. The parameters $C_{1}$ and $S_{2}$, which control the saturation of T-cell proliferation by IL- 2 and TGF- $\beta$ respectively, were calculated from experiments in (Thomas and Massagué, 2005; McKarns and Schwartz, 2005). T-cell proliferation was measured for different concentrations of IL-2, and also in the presence or absence of TGF- $\beta$.

The parameters for the conversion of helper $\mathrm{T}$ cells into Tregs are based on systemic levels of TGF- $\beta$ (Wakefield et al., 1990; Desser et al., 2001) and Treg to T-cell ratios in cancer patients (Liyanage et al., 2002). With the tumor established, the parameter $\alpha_{7}$ is chosen so that Treg ratios are consistent with the data in Liyanage et al. (2002) for a given tumor size.

Cytokine production and clearance rates were calculated from references shown in Table 4 . Concentrations of the cytokine measured in vitro after a given time period were used to determine production rates per cell. These production rates can vary from tumor to tumor; for the present study a 
representative value was chosen.

\section{Results and Discussion}

\subsection{Main Qualitative Behaviors}

For a given set of immune system parameters, the effectiveness of the immune system against the tumor depends on the tumor antigenicity and the tumor growth rate. Figure 3 shows the results of the model for various values of the growth rate and antigenicity. The horizontal axis represents increasing tumor antigenicity, and the vertical axis represents increasing tumor growth rate. The axis labels on the left show the time it would take a tumor to reach $10^{11}$ cells (representing a lethal volume) in the absence of any immune system response (i.e. setting parameter $r_{0}=0$ ). The axis labels on the right show the corresponding values of the control parameter $\gamma$. Long-term behavior of the tumor was determined for the ranges of the two control parameters shown on the axes, using fixed point analysis (described below in section 4.3). The long-term behavior of the tumor has three possible outcomes: tumor removal, considered to occur when the value of $T$ falls below 1 cell; tumor control, which occurs if the tumor is bounded, reaching either a stable fixed point or a stable limit cycle; or unbounded growth. The initial conditions for all simulations was $T_{0}=1$ cell; other cell populations were set to zero, except variables that appear in denominator terms were set to a small value $\left(10^{-5}\right)$ to avoid singularities. Initial conditions for the cytokines were set to zero.

The area marked Removal in Figure 3 represents the tumors that are removed by the immune system. These tumors are slower growing and more antigenic, and the immune system is able to kill the tumor cells faster than the tumor is able to grow. Suppression is limited, since Tregs, TGF- $\beta$ levels, and IL-10 levels never become significant, and the size of the tumor is never large enough to prevent immune cell access to the interior.

The area marked Control represents the tumors that either reach a steady state greater than one cell, or oscillate around a size greater than one cell. The immune system killing rate and the tumor growth rate in Eq. 16 reach an equilibrium. The dotted contour lines in Figure 3 within the Control section show the equilibrium size of the tumor for a given combination of growth rate and antigenicity. For some parameter values, the tumor reaches a stable fixed point, while for other parameters, the tumor oscillates within a stable limit cycle (see section 4.3). The lighter grey section is the 
area in which the tumor reaches a stable limit cycle, and the darker grey region is where the tumor reaches a stable fixed point.

The stable size of the tumor is always between 1 cell and about $10^{8}$ cells. Stable fixed points with tumor sizes greater than $10^{8}$ cells were not observed for any growth rate or antigenicity. This region of stability at a small volume can be viewed as a mechanism by which a tumor can remain dormant for a period. However, it is important to note that the model is deterministic, so that a steady state in the model will persist forever. In the biological situation, conditions are constantly changing, due to tumor cell mutations, health of the host, and other factors. These random effects can destabilize an equilibrium and cause the tumor to begin to grow or be removed.

The area marked Growth in Figure 3 shows the tumors which grow without bound, eventually reaching a detectable size $\left(10^{9}\right.$ cells and greater $)$. The dashed lines in the growth section represent contours of the time needed for the tumor to reach $10^{11}$ cells in size. On the left side of the figure, for $a=10^{-5}$, the contour lines essentially reach the values determined by free tumor growth shown on the left-hand axis, indicating that for antigenicities less than this value, the immune system has no significant effect on tumor growth. As antigenicity is increased, the immune system does slow down tumor growth within the Growth region, up to the optimum value of about $10^{-1.5}$. Further increases in the antigenicity beyond this level will cause the tumor to grow faster than at the optimal antigenicity.

Tumors with high growth rates escape because they grow faster than the immune system can kill them. Of interest is the region of growth to the right of the control peak (point B). Even though the antigenicity is higher than within the control peak, the tumor grows without bound. In a linear system, increasing the antigenicity would result an increased response of the immune system, and therefore more favorable tumor outcome. However, these highly antigenic tumors in the region near point $\mathbf{B}$ escape because they promote a large increase in Treg populations and TGF- $\beta$ levels, and thus suppress the immune system despite the high antigenicity.

\subsection{Immunosuppressive Effects of TGF- $\beta$, Converted Tregs, and IL-10}

Figure 4 shows the long-term behavior of the tumor when TGF- $\beta$ is removed from the system, by setting the value of $S$ to zero. This has two effects on the system: the direct suppressive effects of TGF- $\beta$ are removed, and the conversion of helper cells to Tregs is stopped. These are the two mechanisms responsible for the existence of the optimal antigenicity peak evident 
in Figure 3. When these mechanisms are removed, the fixed point contours continuously increase towards an asymptotic value, rather than displaying a peak. As expected, the control region expands significantly, since much of the immunosuppression has been removed. However, the lower portion of the figure, corresponding to slower growing tumors, is minimally impacted by the change. Tumors which are removed and those which are maintained at a small volume of $T<10^{6}$ cells exhibit very similar dynamics as in Figure 3. This is expected because conversion of helper cells to Tregs depends on TGF- $\beta$, and the level of TGF- $\beta$ at small tumor sizes is negligible.

Figure 5 shows the long-term tumor behavior when only the conversion of helper cells to Tregs by TGF- $\beta$ has been disabled by setting parameter $\alpha_{7}$ to zero. The direct suppressive effects of TGF- $\beta$ remain in effect. The upper Control region has expanded in this figure as well. The peak associated with an optimal antigenicity persists, though shifted to the right about one order of magnitude in antigenicity.

The effect of TGF- $\beta$ suppression on the system is shown in Figure 6, where the direct suppressive effects of TGF- $\beta$ have been disabled by removing the suppressive terms associated with parameters $S_{1}, S_{2}$, and $S_{4}$ in Eqs. (16), (20), (22), and (25). Conversion of helper T cells into Tregs is still active for this figure. The upper Control area is expanded in this case. The existence of an optimal antigenicity persists for tumors that reach a stable size between $10^{6}$ and $10^{9}$ cells. Larger stable tumors can be reached when the antigenicity is high, in contrast to Figures 3 and 5 where these tumors would grow without bound. These high stability points do not exhibit an optimum antigenicity profile. As with Figure 5, the lower portion of the figure for small tumor sizes and removed tumors remains unchanged, due to minimal TGF- $\beta$ suppression at these levels.

For the production rates of IL-10 found in the literature, IL-10 has a minimal suppressive effect in the model. The production rates of IL-10 would need to be increased by over two orders of magnitude to see a difference in suppression comparable to that seen with Tregs and TGF- $\beta$. This is not consistent with experimental evidence showing that for some types of cancer, IL-10 has a significant effect on immunosuppression (Avradopoulos et al., 1997). The secretion of IL-10 in these cancers may be higher than estimated in this study. It is also possible that IL-10 acts locally in a paracrine fashion, rather than systemically, in which case the magnitude of suppression would be underestimated in the model. This question could be further investigated in a spatially realized model. 
The existence of an optimal antigenicity has bearing on immunotherapies which attempt to increase the antigenicity of a tumor (dendritic cell therapy, for example). For slower growing tumors $(\gamma<400)$, increases in antigenicity are wholly beneficial, since this leads to tumor removal. For faster growing tumors, however, there is an optimal antigenicity and increasing beyond this point will result in decreased therapeutic effect by increasing the effective growth rate, and possibly allowing a controlled tumor to grow without bound.

The importance of various suppressive effects at different stages of tumor growth can be quantified. The overall killing rate of tumor cells by effector $\mathrm{T}$ cells is given by the second term of Eq. (16). The unsuppressed rate, $r_{0}$ is altered directly by three factors: the presence of Tregs, the presence of TGF- $\beta$, and the limitations of access to the interior of the tumor due to poor blood vessel perfusion. By calculating the killing rate with and without each of these suppressive effects in place, the relative strength of each effect can be quantified.

The tumors marked at points $\mathbf{A}$ and $\mathbf{B}$ in Figure 3 take the same amount of time to reach $10^{11}$ cells but exhibit different suppression profiles. Figure 7 shows the results of a simulation for a tumor with parameters $\gamma=333$ and $a=10^{-4.2}$, corresponding to point A. Figure 7a shows the populations of tumor cells, effector T cells, and Tregs. With such low antigenicity, the number of $\mathrm{T}$ cells never becomes large enough to fight the tumor effectively. Figure $7 \mathrm{~b}$ shows how the $\mathrm{T}$ cell population is divided between helper cells, effector cells, and Tregs. As the tumor grows, it recruits more Tregs, primarily by producing TGF- $\beta$ which converts the helper cells. Figure $7 \mathrm{c}$ shows the fraction of Tregs that is naturally expanded from the memory population, and the fraction that is derived from helper cells. Figure $7 \mathrm{~d}$ shows the suppressive effects that are acting to prevent tumor cell killing. The solid line represents the relative tumor kill rate due to all suppressive effects, where a value of 1 would indicate that there is no suppression of the killing rate. The three other traces show the contribution to this suppression by Tregs, TGF- $\beta$, and tumor access limitation $\left(T^{*}\right)$. For this growth rate and antigenicity, Tregs exert increasing suppression with increasing tumor size, but the primary reason that the tumor escapes is that the effector $\mathrm{T}$ cell numbers are too low to provide significant tumor cell kill. TGF- $\beta$ suppression is only significant when the tumor is large. Limited access to the interior of the tumor does not significantly affect the immune system response, because tumor to effector cell ratio is large, regardless of access limitations. The effector cells that are present are insufficient to battle the accessible tumor 
cells, regardless of access to the interior.

By contrast, Figure 8 shows the results of the model for a tumor with parameters $\gamma=574$ and $a=10$. This corresponds to the point labeled $\mathbf{B}$ in Figure 3. In this case, the Tregs expand very quickly, and immediately suppress T-cell killing. T-cell numbers are very high, but limited access to the center of the tumor also limits the immune system efficacy, as seen in the suppression plot (Figure 8d). Treg suppression is enhanced by early conversion of helper cells into Tregs. Although the high antigenicity causes large numbers of effector $\mathrm{T}$ cells to develop, there are also large numbers of helper $\mathrm{T}$ cells, which in turn become Tregs through TGF- $\beta$ mediated conversion. This enhances Treg suppression of tumor cell killing.

In order to achieve the optimal immune response, the antigenicity has to be large enough to provoke a significant immune response, but not so large that the suppressive effects overwhelm the cytotoxic ability of effector $\mathrm{T}$ cells.

Tregs and TGF- $\beta$ appear to have the broadest suppressive effect on tumor cell killing, consistent with experimental evidence showing the importance of these suppressive factors (Gorelik and Flavell, 2001; Viehl et al., 2006; Frumento et al., 2006; Kobie and Akporiaye, 2003; Wojtowicz-Praga, 2003), with Tregs particularly acting in the early stages of tumor growth. IL-10 had a limited qualitative effect on tumor outcomes in the model, for the production rates derived from the literature. In all cases, TGF- $\beta$ does not appear to have a significant suppressive effect until the tumor is large, $10^{7}$ cells or more. TGF- $\beta$-induced conversion of helper cells into Tregs also becomes significant primarily above this threshold.

The lack of access to the center of the tumor by the effector cells is a significant factor limiting the tumor cell killing when effector cell numbers are high. This could limit the effectiveness of adoptive immunotherapies which increase T-cell numbers and dendritic cell-based therapies which promote dendritic cell activity, since they cannot easily penetrate the tumor interior, therefore limiting the effect of additional cytotoxic cells.

\subsection{Fixed point analysis}

Numerical simulations using the model show the existence of fixed points, as well as stable limit cycles. These are observed within the biologically reasonable range of values for the variables, specifically tumor sizes between 1 cell and $10^{8}$ cells. Numerical analysis of fixed points was performed. Fixed points were found by using a root solver in Mathematica, and the eigenvalues 
of the Jacobian of the matrix of equations were examined to determine the type and stability of the fixed points.

Figure 9 shows bifurcation diagrams for several values of the antigenicity, using the tumor growth rate as the bifurcation parameter. The vertical axis shows the number of tumor cells on a log scale. For a tumor with low antigenicity (Figure 9a), the stable branch (solid line) and the unstable branch (dotted line) annihilate at a low growth rate. Tumors which grow slower than this value will reach the stable equilibrium branch, while tumors that grow faster will escape without bound, since the zero solution is unstable.

As the antigenicity is increased (Figure 9b), the stable branch moves lower and becomes a stable limit cycle (dashed line) for a certain range of growth rates. Tumors in this parameter regime exhibit oscillatory behavior. It is possible that the oscillations may cause the tumor to be smaller than one cell at the minima of each cycle, therefore effectively curing the tumor, but this effect does not significantly affect the analysis of the tumor dynamics. The oscillations are small enough that the Removal region of Figure 3 would be only slightly larger than shown if the oscillatory nature of these tumors was considered when determining if the tumor was cured or controlled.

A further increase in antigenicity (Figure 9c) leads to the development of two lobes in the bifurcation diagram. Slower growing tumors will reach the lower stable branch. Tumors growing faster than the growth rate at the lower bifurcation point will reach the upper stable branch instead.

The lower branch continues to move descend on the diagram with increasing antigenicity, and eventually will cross into the cure boundary of one tumor cell (Figure 9d). Until this level antigenicity is reached, there is no mechanism for complete tumor removal by the immune system.

This behavior continues with increasing antigenicity until the upper stable lobe no longer extends beyond the lower lobe (Figure 9e). Since the lower stable branch has moved below the cure boundary of one tumor cell, and the upper stable branch is inaccessible from an initial condition of one cell, the dynamics only allow for two options. If the growth rate is less than the lower lobe bifurcation point (approximately $\gamma=435$ ) then the tumor will be removed. If the growth rate is higher, it will grow without bound. There is no stable control of the tumor, since the fixed points are either below one cell or inaccessible to a growing tumor. Mathematically, it is of interest to note that tumors which are removed do not go to zero size in the simulation. If the condition for tumor cure is removed from the simulation, all cured tumors would reach a stable fixed point or limit cycle with values less than 
one cell.

Further increases in antigenicity beyond Figure 9f do not significantly affect the dynamics. Tumors either grow without bound, or are removed by the immune system. The bifurcation point stabilizes with respect to antigenicity, as seen by the horizontal boundary between the Removal and Growth regions on the right side of Figure 3. This phenomenon occurs because the immune system saturates at such high antigenicities, and therefore only the growth rate determines the long-term behavior of the tumor.

If the analysis is performed with different initial conditions $\left(T_{0}=10^{5}\right.$, for example), then the upper stable branch that is inaccessible to a small tumor at high antigenicities may be reached. This observation may have an implication for experiments where tumors are implanted into a host, but does not affect the dynamics of tumors started from one cell.

\subsection{Behavior of small tumors in the limiting case}

Since a tumor behaves differently at small sizes than at large sizes, both with respect to growth law and immune system accessibility, the model can be examined in the limiting case as $T<<T_{1}$, where $T_{1}$ is the transition size between growth laws.

For small tumors, TGF- $\beta$ production is negligible, so Eq. (16) can be written as

$$
\dot{T}=\gamma_{1} T-\frac{r_{0} T}{\left(1+k_{2} \frac{T}{E}\right)} \cdot \frac{1}{\left(1+k_{3} \frac{R}{E}\right)} .
$$

The fixed point of this equation occurs when $\dot{T}=0$, or when

$$
\frac{r_{0}}{\gamma\left(T_{1}\right)^{m-1}}=\left(1+k_{2} \frac{T}{E}\right)\left(1+k_{3} \frac{R}{E}\right),
$$

where $\gamma_{1}$ was replaced using Eq. (28). The primary interest of modeling cancer is to find regimes where the tumor diminishes in size. For small tumors, this occurs whenever

$$
\frac{r_{0}}{\gamma\left(T_{1}\right)^{m-1}}>\left(1+k_{2} \frac{T}{E}\right)\left(1+k_{3} \frac{R}{E}\right) .
$$

Since the right-hand side of Eq. (32) is always greater than 1, if the exponential growth rate $\gamma\left(T_{1}\right)^{m-1}$ is higher than the killing rate $r_{0}$, there is no possibility that the immune system can reduce the tumor burden. For the 
parameters used in this analysis given in Table 1, tumors with growth rates $\gamma>900$ will always grow to a large size.

For tumors with $\gamma<900$, the behavior of the tumor will depend on the right-hand side of Eq. (32). A failure of the immune system to clear a tumor is either due to insufficient numbers of effector cells for a given tumor size, leading to a large value for the first term on the right-hand side, or high Treg ratios, leading to a high value for the second term. These are the two mechanisms represented respectively by points $\mathbf{A}$ and $\mathbf{B}$ in Figure 3. The tumor with low antigenicity at point $\mathbf{A}$ escapes because of decreased T-cell stimulation, so the first term on the right side of Eq. (32) dominates. For the highly antigenic tumor at point $\mathbf{B}$, the $T / E$ ratio is very low, since $T$ cells are highly stimulated by the tumor. However, the second term begins to dominate, since Tregs ratios become saturated with increased antigenicity. In between these two regimes lies the optimal region, where the right hand side of Eq. (32) is minimized, and the killing rate has the greatest advantage over the growth rate.

Figure 10 shows solution curves of Eq. (31) assuming that the ratio $R / E$ remains constant. For any given constant ratio $R / E$, a choice of the parameter $\gamma$ and asymptotic ratio $T / E$ characterizes a unique point $(\log (E / T), \gamma)$. If this point is above the corresponding curve, the dynamics of the system will lead to unbounded tumor growth whereas a point below the curve will lead to tumor removal.

As discussed in section 4.1, for high antigenicities there is sharp transition between tumors that are cured and tumors that grow to a large size, greater than $T_{1}=10^{6}$ cells. This is because $E / T$ ratios are large in this regime, making the first term on the right side of Eq. (32) insignificant. The dynamic of the tumor is therefore determined by the ratio of Tregs. For high values of antigenicity, the ratio of Tregs to effector $\mathrm{T}$ cells will approach 0.1 , since Treg conversion from helper cells is negligible when a tumor is small. The contour for $R / E=0.1$ in Figure 10 approaches a $\gamma$ value of about 435 for high $E / T$ ratios (dotted line in Figure 10). This effectively sets an upper limit for the growth rate of a tumor that can be fully removed or controlled at a small size by the immune system, and corresponds to the sharp boundary between the Removal and Growth areas on the right side of Figure 3. It also matches value the lower bifurcation point in Figure 9f. 


\subsection{Comparison with other models}

A number of models of tumor-immune interactions have been published in the literature. Recent models that examine T-cell response have focused on specific aspects of the interactions. Arciero et al. (2004) examined the effects of TGF- $\beta$ suppression on T-cell response. The mathematical model showed that tumors producing TGF- $\beta$ were more likely to escape the immune system, for reasonable parameter values. The model of de Pillis et al. (2005) examined T-cell interactions with natural killer cells. Multiple regimes of tumor growth and removal were found. However, the model did not include any suppressive effects. Leon et al. (2007) have modeled Tregs and their effect on T-cell response. They found two modes of unbounded tumor growth, one where the Tregs exert suppression on the effector cells, and one where the Tregs have a minimal effect. These regimes, while qualitatively similar to the dynamics observed for points $\mathbf{A}$ and $\mathbf{B}$, are not distributed in the growthantigenicity parameter space in the same way that was found in this paper. The approach to Treg expansion in that study is different than the method used in the present paper, and other important suppressive elements such as TGF- $\beta$ are not included. In addition, the conversion of Helper T-cells into Tregs was not addressed in the Leon model.

\section{Discussion}

The mathematical model developed here combines the primary suppressive mechanisms involved in the T-cell response and quantifies the importance of the different suppressive effects at different stages of tumor growth. Regulatory T-cells are shown to have significant effect on suppression at all stages, while TGF- $\beta$ has an effect that scales with tumor size. In addition, the limitations of access to the interior of a large tumor can be a significant factor in preventing immune system killing of the tumor cells. IL-10 had the least significant suppressive effect, although tumors producing high IL-10 values may exhibit different dynamics.

The immune response provoked by a tumor varies widely according to its growth rate and antigenicity. Although a simple immune response model would predict that greater antigenicity would always lead to greater immune response against the tumor cells, our model shows that there is an optimal antigenicity for fast growing tumors. If a fast growing tumor is minimally antigenic, then there is only a small immune response; if it is highly antigenic, there may be a large immune response, but also a large suppressive response 
by Tregs and TGF- $\beta$. In between these two regimes, the immune response may be large enough to affect the tumor, but avoid excessive promotion of Tregs and other suppressive effects.

Extensions of this model have been made by the authors to include the effects of chemotherapy (Robertson-Tessi et al., 2010) in combination with the immune response. It would also be of interest to expand the scope of the model to include a spatial element, and also compartmentalize the various interactions between cells. At the moment, the model simulates a well mixed system, except for the access limitations due to vasculature already described. Since T-cell priming and expansion happens away from the tumor in the lymph nodes, this additional effect should affect the dynamics of the system. In addition, suppressive effects would be different at the tumor site than in the lymph nodes and Treg populations in the tumor may be much larger than in the lymphatic system. However, estimating the parameters for these more detailed interactions presents a challenge beyond the scope of this initial model.

Understanding the key processes in tumor-immune interactions will be critical to develop effective therapies, both in terms of targets, and optimization of dosing and schedule. The model presented here takes a step towards demonstrating the effects of Tregs and TGF- $\beta$ on various classes of tumors, and further extensions of the model can be used to examine how treatments interact with this system.

\section{Acknowledgements}

This publication is based on work supported by Award No. KUK-C1-01304, made by King Abdullah University of Science and Technology (KAUST), and based in part upon work supported by the National Science Foundation under grants DMS-0907773 (AG). AG is a Wolfson/Royal Society Merit Award Holder. This publication is based on work supported by the ARCS Foundation, NSF-VIGRE, and the BIO5 Institute at the University of Arizona (MRT).

\section{References}

Alleva, D., Burger, C., Elgert, K., 1994. Tumor-induced regulation of suppressor macrophage nitric oxide and TNF-alpha production. Role of tumorderived IL-10, TGF- $\beta$, and prostaglandin E2. J. Immunol. 153, 1674. 
Arciero, J., Jackson, T., Kirschner, D., 2004. A mathematical model of tumor-immune evasion and siRNA treatment. Discret. Contin. Dyn. S. Ser. B 4, 39-58.

Avradopoulos, K., Mehta, S., Blackinton, D., Wanebo, H., 1997. Interleukin10 as a possible mediator of immunosuppressive effect in patients with squamous cell carcinoma of the head and neck. Annals Surg. Oncol. 4, 184-190.

Chen, L., McGowan, P., Ashe, S., Johnston, J., Li, Y., Hellstrom, I., Hellstrom, K., 1994. Tumor immunogenicity determines the effect of B7 costimulation on T-cell mediated tumor immunity. J. Exp. Med. 179, 523.

Chouaib, S., Asselin-Paturel, C., Mami-Chouaib, F., Caignard, A., Blay, J., 1997. The hosttumor immune conflict: from immunosuppression to resistance and destruction. Immunol. Today 18, 493-497.

Danforth Jr, D., Sgagias, M., 1996. Tumor necrosis factor alpha enhances secretion of transforming growth factor $\beta 2$ in MCF-7 breast cancer cells. Clin. Cancer Res. 2, 827.

De Boer, R., Perelson, A., 1994. T-cell repertoires and competitive exclusion. J. Theor. Biol. 169, 375-375.

Desser, L., Holomanova, D., Zavadova, E., Pavelka, K., Mohr, T., Herbacek, I., 2001. Oral therapy with proteolytic enzymes decreases excessive TGF- $\beta$ levels in human blood. Cancer Chemoth. Pharm. 47, 10-15.

Dudley, M., Wunderlich, J., Robbins, P., Yang, J., Hwu, P., Schwartzentruber, D., Topalian, S., Sherry, R., Restifo, N., Hubicki, A., et al., 2002. Cancer regression and autoimmunity in patients after clonal repopulation with antitumor lymphocytes. Science 298, 850.

Dunn, G., Bruce, A., Ikeda, H., Old, L., Schreiber, R., 2002. Cancer immunoediting: from immunosurveillance to tumor escape. Nat. Immunol. 3, 991-998.

Dunn, G., Koebel, C., Schreiber, R., 2006. Interferons, immunity and cancer immunoediting. Nat. Rev. Immunol. 6, 836-848. 
Fitzpatrick, D., Bielefeldt-Ohmann, H., Himbeck, R., Jarnicki, A., Marzo, A., Robinson, B., 1996. Tumour-induced immunosuppression and autocrine growth: use of inducible anti TGF- $\beta$ antisense RNA transfectants in a mesothelioma model. Lung Cancer 15, 260-260.

Friberg, S., Mattson, S., 1997. On the growth rates of human malignant tumors: implications for medical decision making. J. Surg. Oncol. 65, 284-297.

Frumento, G., Piazza, T., Di Carlo, E., Ferrini, S., 2006. Targeting tumorrelated immunosuppression for cancer immunotherapy. Endocr. Metab. Immune. Disord. Drug Targets 6, 223-237.

Gastl, G., Abrams, J., Nanus, D., Oosterkamp, R., Silver, J., Liu, F., Chen, M., Albino, A., Bander, N., 1993. Interleukin-10 production by human carcinoma cell lines and its relationship to interleukin-6 expression. Int. J. Cancer 55, 96-96.

Gorelik, L., Flavell, R., 2001. Immune-mediated eradication of tumors through the blockade of transforming growth factor $\beta$ signaling in $\mathrm{T}$ cells. Nat. Med. 7, 1118-1122.

Hart, D., Shochat, E., Agur, Z., 1998. The growth law of primary breast cancer tumors as inferred from mammography screening trials. Br. J. Cancer 78, 382-387.

Hellstrbm, K., Hellstrom, I., 1991. Principles of tumor immunity: tumor antigens, in: De Vita, V., Hellman, S., Rosenberg, S. (Eds.), Biologic Therapy of Cancer: Principles and Practices. J.B. Lippincott, Philadelphia, pp. $35-52$.

Hellstrom, K., Hellstrom, I., 1974. Lymphocyte-mediated cytotoxicity and blocking serum activity to tumor antigens. Adv. Immunol. 18, 209-277.

Hsieh, C., Chen, D., Hwang, L., 2000. Tumor-induced immunosuppression: a barrier to immunotherapy of large tumors by cytokine-secreting tumor vaccine. Human Gene Ther. 11, 681-692.

Iwashita, I., Ueyama, T., Iwashita, A., Kawamoto, K., Kitagawa, S., Motooka, M., Utsunomiya, T., Masuda, K., 1998. Natural history of colorectal carcinoma: can the tumor volume doubling time be predicted by 
radiologic findings or immunohistochemical variables? J. Surg. Oncol. 68, 215.

Jackson, C., Talpos, G., Block, M., Norum, R., Lloyd, R., Tashjian, A., 1984. Clinical value of tumor doubling estimations in multiple endocrine neoplasia type II. Surgery 96, 981-987.

Jain, R., Ward-Hartley, K., 1984. Tumor blood flow: characterization, modifications, and role in hyperthermia. IEEE Trans. Sonics Ultrason. 31, $504-526$.

Jarnicki, A., Lysaght, J., Todryk, S., Mills, K., 2006. Suppression of antitumor immunity by IL-10 and TGF- $\beta$-producing $\mathrm{T}$ cells infiltrating the growing tumor: influence of tumor environment on the induction of $\mathrm{CD} 4^{+}$ and $\mathrm{CD} 8^{+}$regulatory T cells. J. Immunol. 177, 896.

Kirschner, D., Panetta, J., 1998. Modeling immunotherapy of the tumorimmune interaction. J. Math. Biol. 37, 235-252.

Kobie, J., Akporiaye, E., 2003. Immunosuppressive role of transforming growth factor beta in breast cancer. Clin. Appl. Immunol. Rev. 3, 277287.

Konrad, M., Hemstreet, G., Hersh, E., Mansell, P., Mertelsmann, R., Kolitz, J., Bradley, E., 1990. Pharmacokinetics of recombinant interleukin 2 in humans. Cancer Res. 50, 2009.

Kripke, M., 1974. Antigenicity of murine skin tumors induced by ultraviolet light. J. Natl. Cancer Inst. 53, 1333.

Kuznetsov, V., Makalkin, I., Taylor, M., Perelson, A., 1994. Nonlinear dynamics of immunogenic tumors: Parameter estimation and global bifurcation analysis. Bull. Math. Biol. 56, 295-321.

Larmonier, N., Marron, M., Zeng, Y., Cantrell, J., Romanoski, A., Sepassi, M., Thompson, S., Chen, X., Andreansky, S., Katsanis, E., 2007. Tumorderived $\mathrm{CD} 4{ }^{+} \mathrm{CD} 25^{+}$regulatory T-cell suppression of dendritic cell function involves TGF- $\beta$ and IL-10. Cancer Immunol. Immunoth. 56, 48-59.

Le, T., Leung, L., Carroll, W., Schibler, K., 1997. Regulation of interleukin10 gene expression: possible mechanisms accounting for its upregulation 
and for maturational differences in its expression by blood mononuclear cells. Blood 89, 4112.

Leon, K., Garcia, K., Carneiro, J., Lage, A., 2007. How regulatory CD25+ $\mathrm{CD}^{+} \mathrm{T}$ cells impinge on tumor immunobiology? On the existence of two alternative dynamical classes of tumors. J. Theor. Biol. 247, 122-137.

Liu, K., Waskow, C., Liu, X., Yao, K., Hoh, J., Nussenzweig, M., 2007a. Origin of dendritic cells in peripheral lymphoid organs of mice. Nat. Immunol. $8,578-583$.

Liu, V., Wong, L., Jang, T., Shah, A., Park, I., Yang, X., Zhang, Q., Lonning, S., Teicher, B., Lee, C., 2007b. Tumor evasion of the immune system by converting $\mathrm{CD} 4^{+} \mathrm{CD} 25^{-} \mathrm{T}$ cells into $\mathrm{CD} 4^{+} \mathrm{CD} 25^{+} \mathrm{T}$ regulatory cells: role of tumor-derived TGF- $\beta$. J. Immunol. 178, 2883.

Liyanage, U., Moore, T., Joo, H., Tanaka, Y., Herrmann, V., Doherty, G., Drebin, J., Strasberg, S., Eberlein, T., Goedegebuure, P., et al., 2002. Prevalence of regulatory $\mathrm{T}$ cells is increased in peripheral blood and tumor microenvironment of patients with pancreas or breast adenocarcinoma. J. Immunol. 169, 2756.

Lotze, M., Frana, L., Sharrow, S., Robb, R., Rosenberg, S., 1985. In vivo administration of purified human interleukin 2. I. Half-life and immunologic effects of the Jurkat cell line-derived interleukin 2. J. Immunol. 134, 157.

McKarns, S., Schwartz, R., 2005. Distinct effects of TGF- $\beta 1$ on CD4 ${ }^{+}$ and $\mathrm{CD} 8^{+}$T-cell survival, division, and IL-2 production: a role for T-cell intrinsic Smad3. J. Immunol. 174, 2071.

Mempel, T., Henrickson, S., Von Andrian, U., 2004. T-cell priming by dendritic cells in lymph nodes occurs in three distinct phases. Nature 427, 154-159.

Norton, L., 1988. A Gompertzian model of human breast cancer growth. Cancer Res. 48, 7067-71.

Peer, P., Van Dijck, J., Verbeek, A., Hendriks, J., Holland, R., 1993. Agedependent growth rate of primary breast cancer. CA Cancer J. Clin. 71, $3547-3551$. 
Piccirillo, C., Letterio, J., Thornton, A., McHugh, R., Mamura, M., Mizuhara, H., Shevach, E., 2002. CD4 ${ }^{+} \mathrm{CD} 25^{+}$regulatory T cells can mediate suppressor function in the absence of transforming growth factor $\beta 1$ production and responsiveness. J. Exp. Med. 196, 237.

Piccirillo, C., Shevach, E., 2001. Cutting edge: control of $\mathrm{CD}^{+} \mathrm{T}$ cell activation by $\mathrm{CD}^{+}{ }^{+} \mathrm{CD} 25^{+}$immunoregulatory cells. J. Immunol. 167, 1137.

de Pillis, L., Radunskaya, A., Wiseman, C., 2005. A validated mathematical model of cell-mediated immune response to tumor growth. Cancer Res. 65,7950 .

Robertson-Tessi, M., El-Kareh, A., Goriely, A., 2010. A mathematical model of tumor-immune interactions with chemotherapy and immunotherapy. in preparation .

Roitt, I., Delves, P., 2001. Roitt's essential immunology. Blackwell Science Inc.: Oxford.

Rosenberg, S., Lotze, M., 1986. Cancer immunotherapy using interleukin-2 and interleukin-2-activated lymphocytes. Ann. Rev. Immunol. 4, 681-709.

Rosenberg, S., Spiess, P., Lafreniere, R., 1986. A new approach to the adoptive immunotherapy of cancer with tumor-infiltrating lymphocytes. Science $233,1318$.

Setoguchi, R., Hori, S., Takahashi, T., Sakaguchi, S., 2005. Homeostatic maintenance of natural Foxp $3^{+} \mathrm{CD} 25^{+} \mathrm{CD} 4^{+}$regulatory $\mathrm{T}$ cells by interleukin (IL)-2 and induction of autoimmune disease by IL-2 neutralization. J. Exp. Med. 201, 723.

Skog, A., Wadhwa, M., Hassan, M., Gharizadeh, B., Bird, C., Ragnhammar, P., Thorpe, R., Mellstedt, H., 2001. Alteration of interleukin 2 (IL-2) pharmacokinetics and function by IL-2 antibodies induced after treatment of colorectal carcinoma patients with a combination of monoclonal antibody 17-1A, granulocyte macrophage colony-stimulating factor, and IL-2. Clin. Cancer Res. 7, 1163. 
Smith, C., Wilson, N., Waithman, J., Villadangos, J., Carbone, F., Heath, W., Belz, G., 2004. Cognate CD4 ${ }^{+} \mathrm{T}$ cell licensing of dendritic cells in CD8 ${ }^{+}$T-cell immunity. Nat. Immunol. 5, 1143-1148.

Steele, G., 1977. Growth kinetics of tumors. Clarendon: Oxford.

Taga, K., Mostowski, H., Tosato, G., 1993. Human interleukin-10 can directly inhibit T-cell growth. Blood 81, 2964.

Tannock, I., Lee, C., Tunggal, J., Cowan, D., Egorin, M., 2002. Limited penetration of anticancer drugs through tumor tissue. Clin. Cancer Res. 8,878 .

Taylor, J., Fahey, J., Detels, R., Giorgi, J., 1989. CD4 percentage, CD4 number, and CD4:CD8 ratio in HIV infection: which to choose and how to use. J. Acquired Immune Defic. Syndromes 2, 114.

Thomas, D., Massagué, J., 2005. TGF- $\beta$ directly targets cytotoxic T-cell functions during tumor evasion of immune surveillance. Cancer Cell 8, 369-380.

Vaage, J., 1971. Concomitant immunity and specific depression of immunity by residual or reinjected syngeneic tumor tissue. Cancer Res. 31, 1655.

Viehl, C., Moore, T., Liyanage, U., Frey, D., Ehlers, J., Eberlein, T., Goedegebuure, P., Linehan, D., 2006. Depletion of $\mathrm{CD}^{+} \mathrm{CD}^{+} 5^{+}$regulatory $\mathrm{T}$ cells promotes a tumor-specific immune response in pancreas cancer-bearing mice. Ann. Surg. Oncol. 13, 1252-1258.

Villunger, A., Strasser, A., 1999. The great escape: is immune evasion required for tumor progression? Nat. Med. 5, 874-875.

Vukmanovic-Stejic, M., Zhang, Y., Cook, J., Fletcher, J., McQuaid, A., Masters, J., Rustin, M., Taams, L., Beverley, P., Macallan, D., et al., 2006. Human CD4 ${ }^{+}$CD25 ${ }^{h i}$ Foxp3 regulatory T cells are derived by rapid turnover of memory populations in vivo. J. Clin. Invest. 116, 2423-2433.

de Waal Malefyt, R., Yssel, H., De Vries, J., 1993. Direct effects of IL-10 on subsets of human $\mathrm{CD} 4^{+} \mathrm{T}$ cell clones and resting T cells. Specific inhibition of IL-2 production and proliferation. J. Immunol. 150, 4754. 
Wakefield, L., Winokur, T., Hollands, R., Christopherson, K., Levinson, A., Sporn, M., 1990. Recombinant latent transforming growth factor $\beta 1$ has a longer plasma half-life in rats than active transforming growth factor $\beta 1$, and a different tissue distribution. J. Clin. Invest. 86, 1976.

Wimmer, C., Rentsch, M., Crispin, A., Illner, W., Arbogast, H., Graeb, C., Jauch, K., Guba, M., 2007. The janus face of immunosuppression - de novo malignancy after renal transplantation: the experience of the Transplantation Center Munich. Kidney Int. 71, 1271-1278.

Wojtowicz-Praga, S., 2003. Reversal of tumor-induced immunosuppression by TGF- $\beta$ inhibitors. Invest. New Drugs $21,21-32$.

Yates, A., Callard, R., 2001. Cell death and the maintenance of immunological memory. Discret. Contin. Dyn. S. 1, 43-59. 


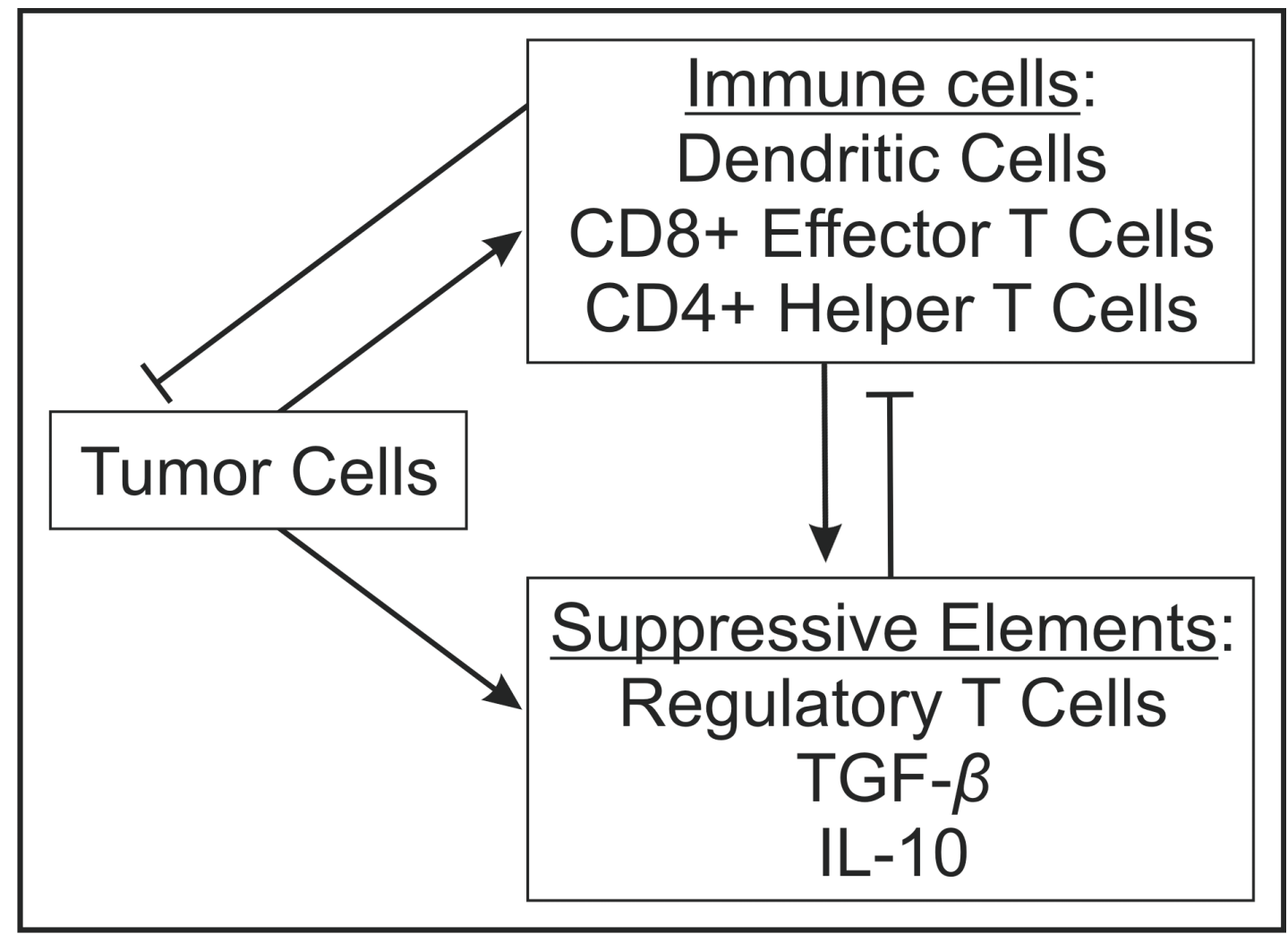

Figure 1: The interactions between a tumor and the immune system. The tumor creates an immune response from $\mathrm{T}$ cells, which can then attack the tumor. At the same time, the tumor promotes immunosuppressive factors, which damp the immune response. 


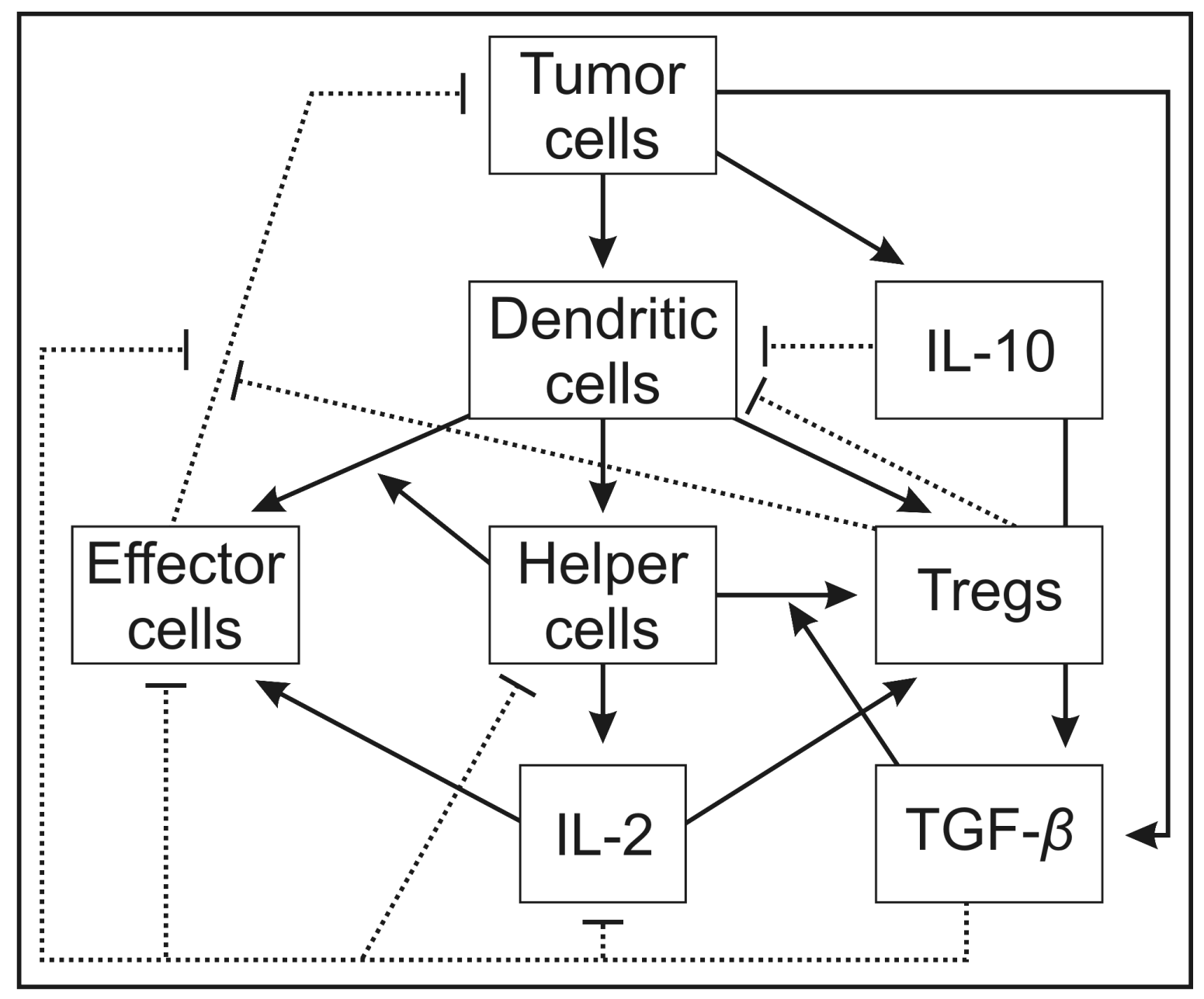

Figure 2: A diagram showing the interactions that are used in the model. Dotted lines show suppressive mechanisms. 


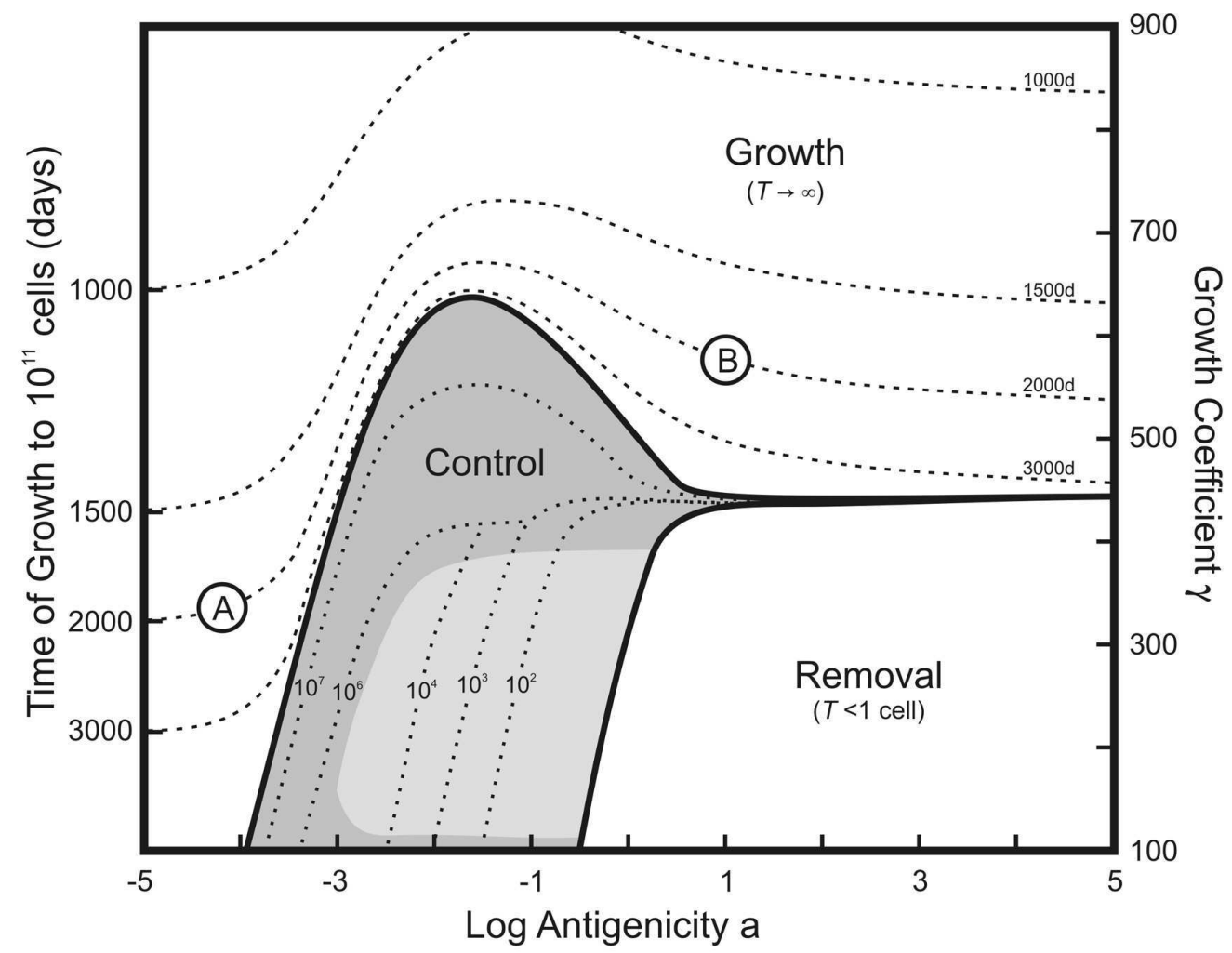

Figure 3: The long term behavior of a tumor. Control parameters are the antigenicity $a$, on the horizontal axis, and tumor growth rate $\gamma$, on the right-hand vertical axis. The left-hand vertical axis shows the time it takes the tumor to reach $10^{11}$ cells in the absence of any immune system killing. The area marked Growth consists of tumors which grow without bound, Removal denotes complete cure of the tumor by the immune system (i.e., $T$ goes below one cell), and Control denotes maintenance of the tumor at either a stable fixed point or a stable limit cycle. The dashed lines in the Growth area show the time it takes for the tumor to reach $10^{11}$ cells. The dotted lines in the Control area show the value of the fixed point. The lighter grey area in the Control section denotes tumors which display a stable limit cycle, and contours passing through this region give the value of the central unstable fixed point. The darker grey area accounts for tumors which grow to stable fixed points. Circled letters refer to simulations shown in Figures 7 and 8. 


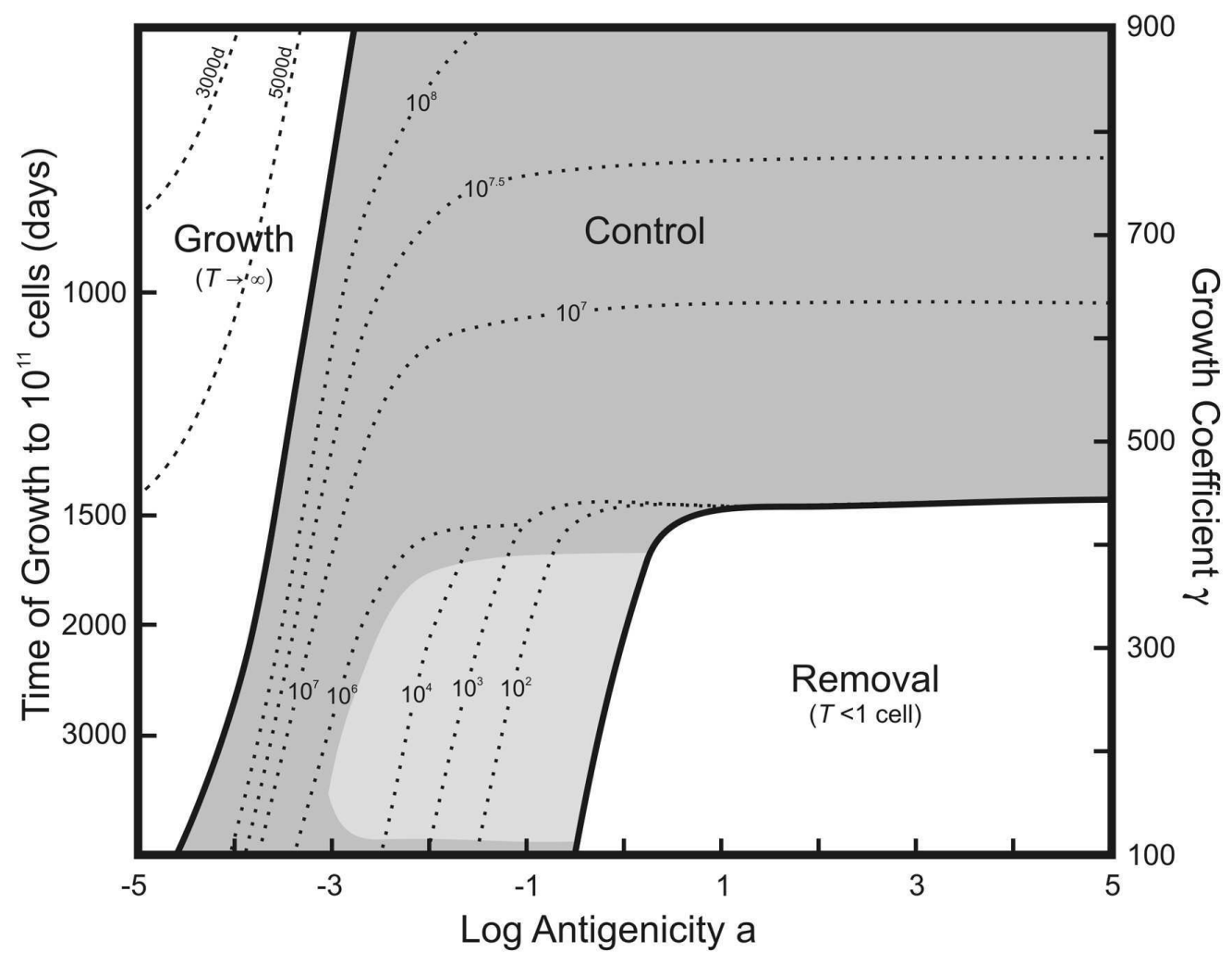

Figure 4: Tumor responses to the immune system, in the absence of TGF- $\beta(S=0)$. In contrast with Figure 3, an optimal antigenicity no longer exists. See caption from Figure 3 for explanation of regions. 


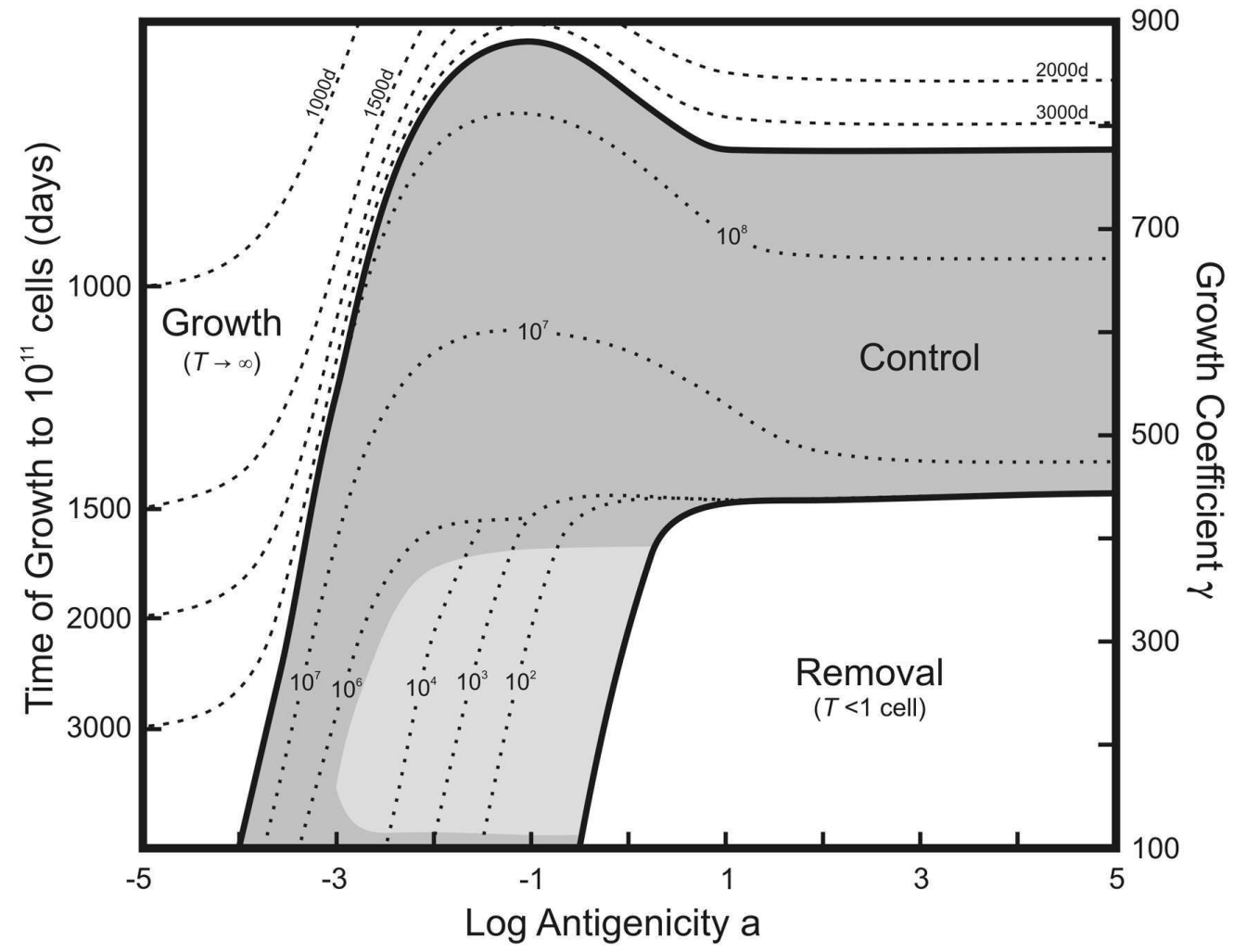

Figure 5: Tumor responses to the immune system, without conversion of helper cells to Tregs (parameter $\alpha_{7}=0$ ). See caption from Figure 3 for explanation of regions. 


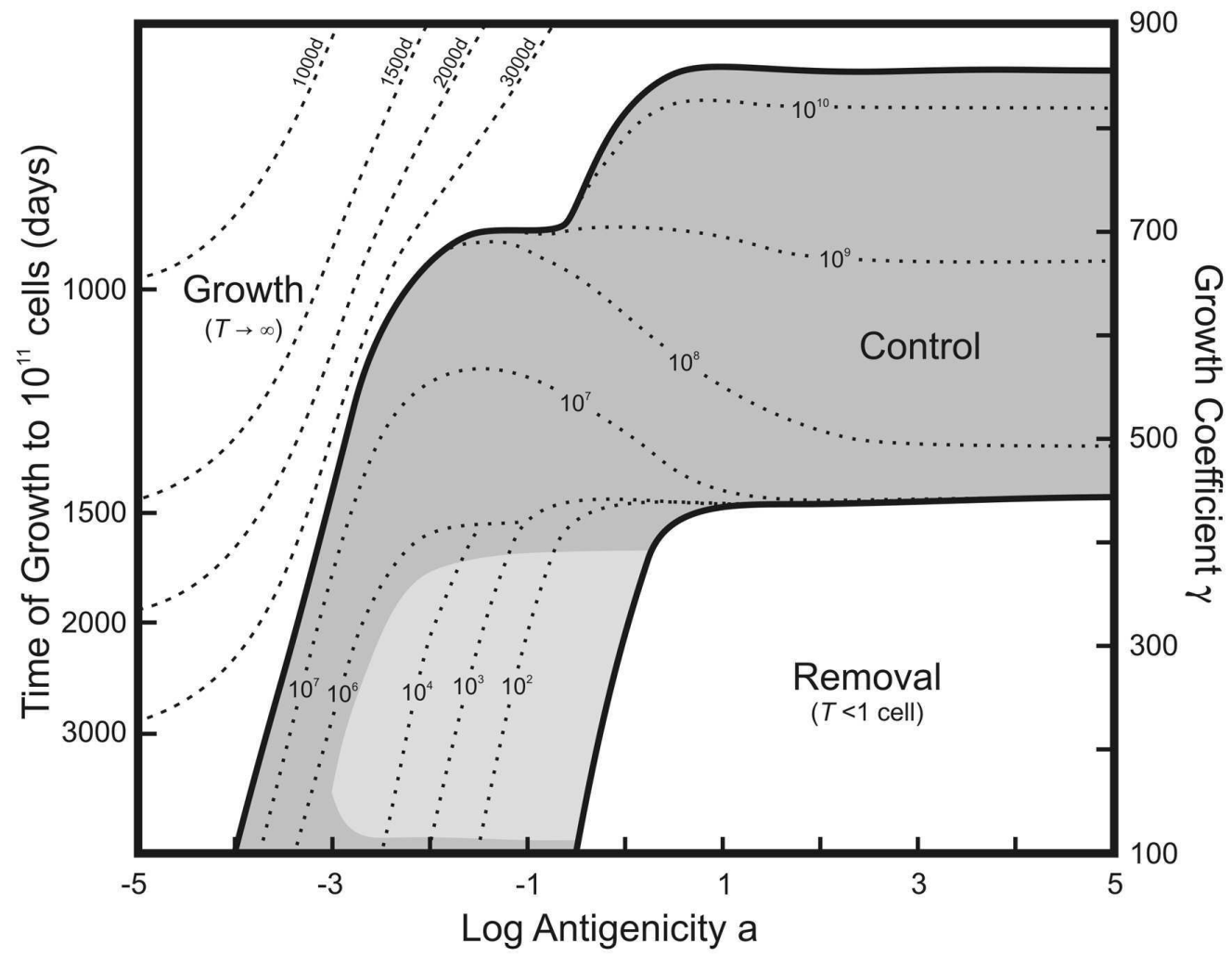

Figure 6: Tumor responses to the immune system, without the direct effects of TGF- $\beta$ suppression. Conversion of helper T cells to Tregs remains active. See caption for Figure 3 from explanation of regions. 

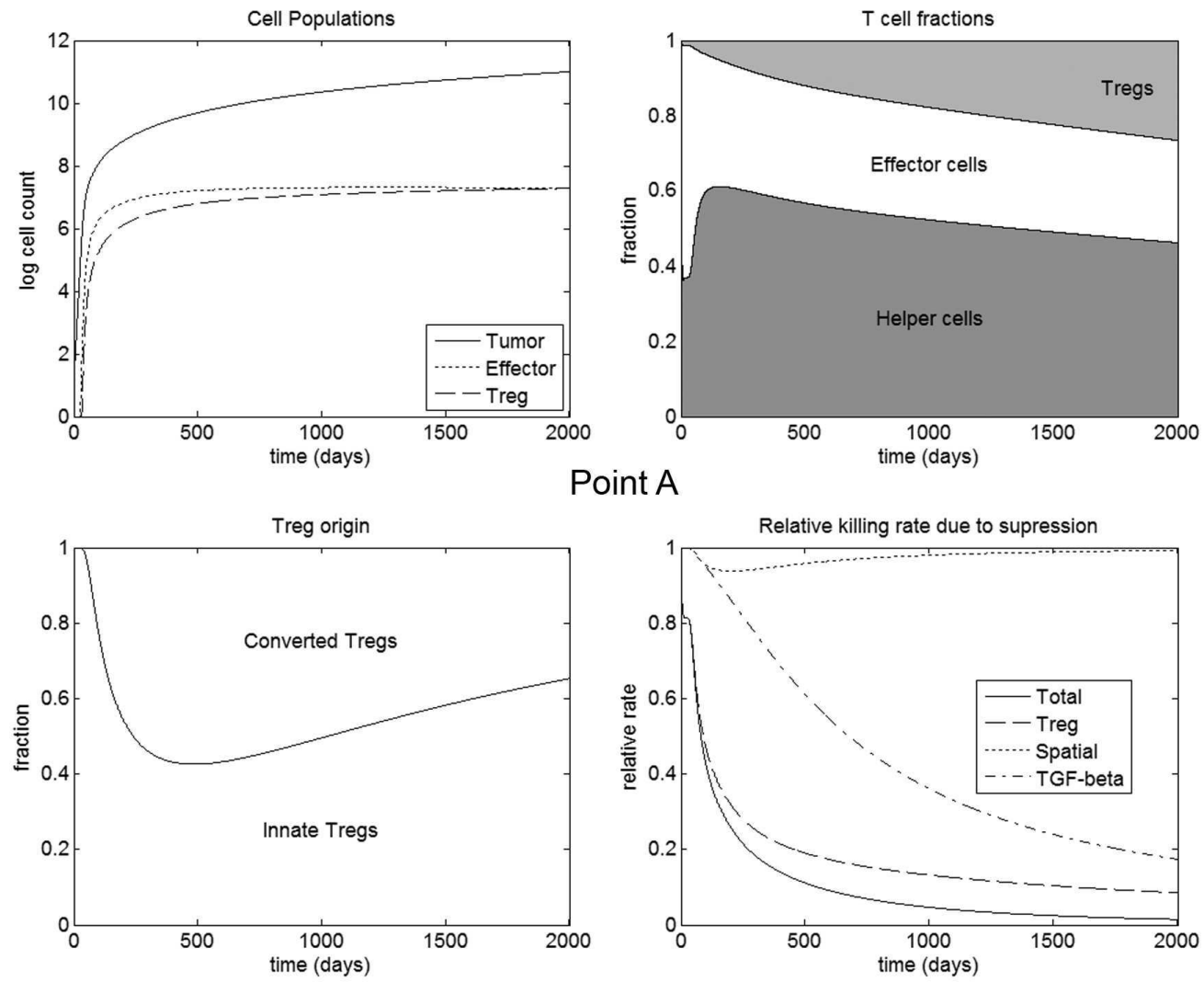

Figure 7: Simulation of tumor growth for $a=10^{-4.2}$ and $\gamma=333$, corresponding to point A in figure 3. a) Cell populations over time. b) T cell fractions. c) Treg origination. d) Suppression of tumor cell killing rate. 

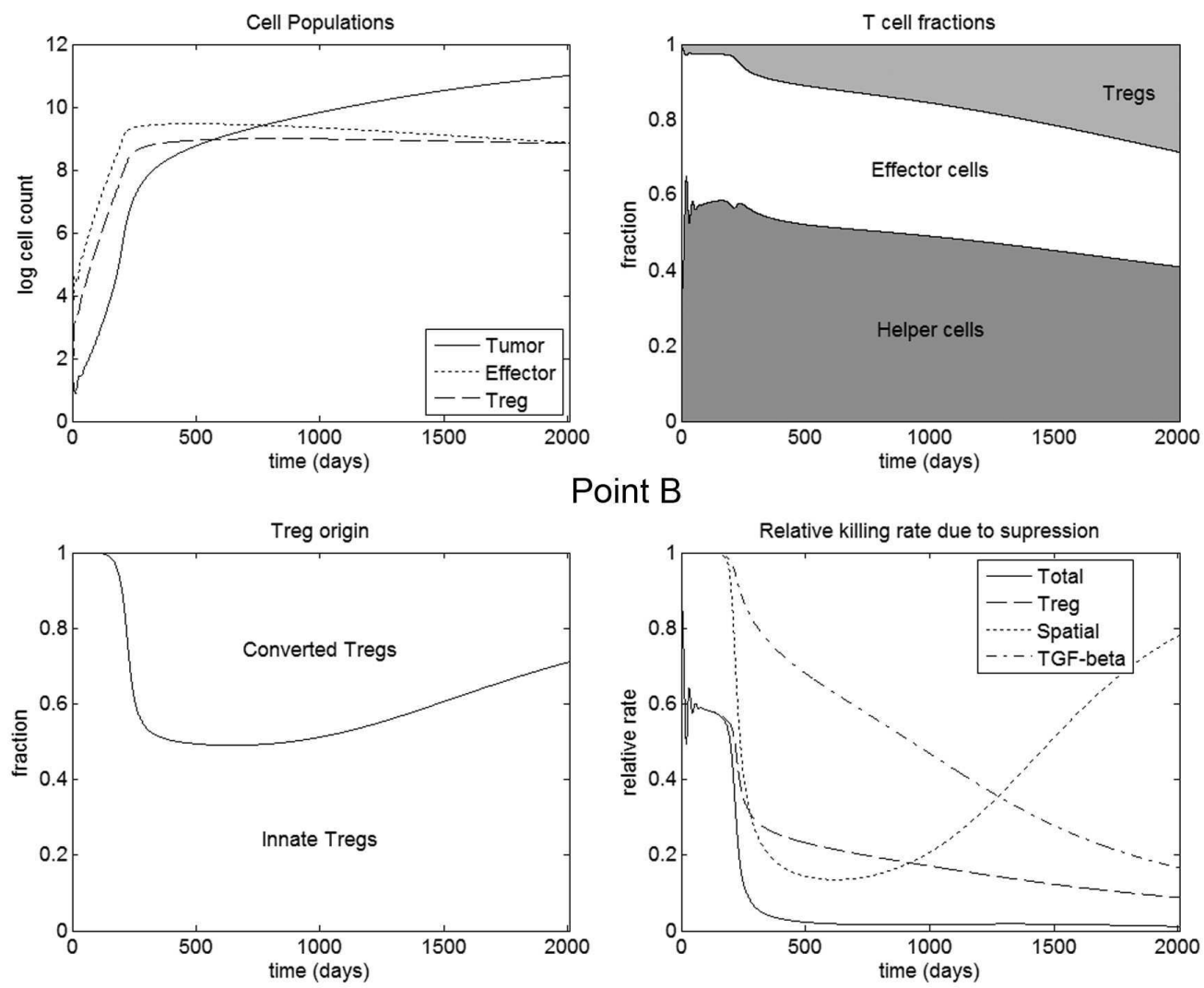

Point B

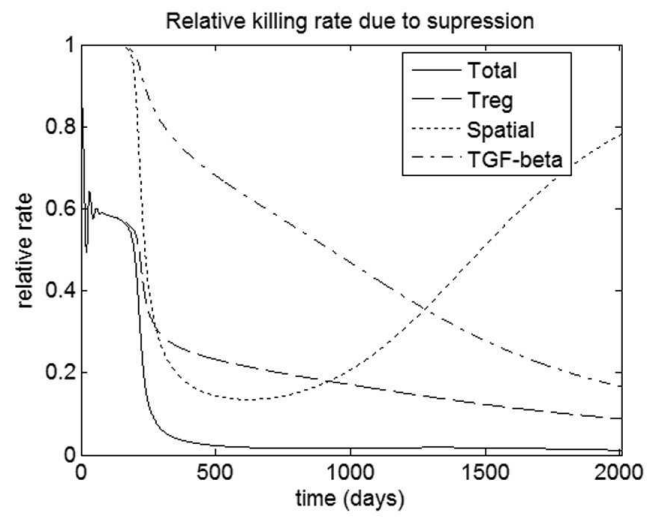

Figure 8: Simulation of tumor growth for $a=10$ and $\gamma=574$, corresponding to point $\mathbf{B}$ in figure 3. a) Cell populations over time. b) T cell fractions. c) Treg origination. d) Suppression of tumor cell killing rate. 


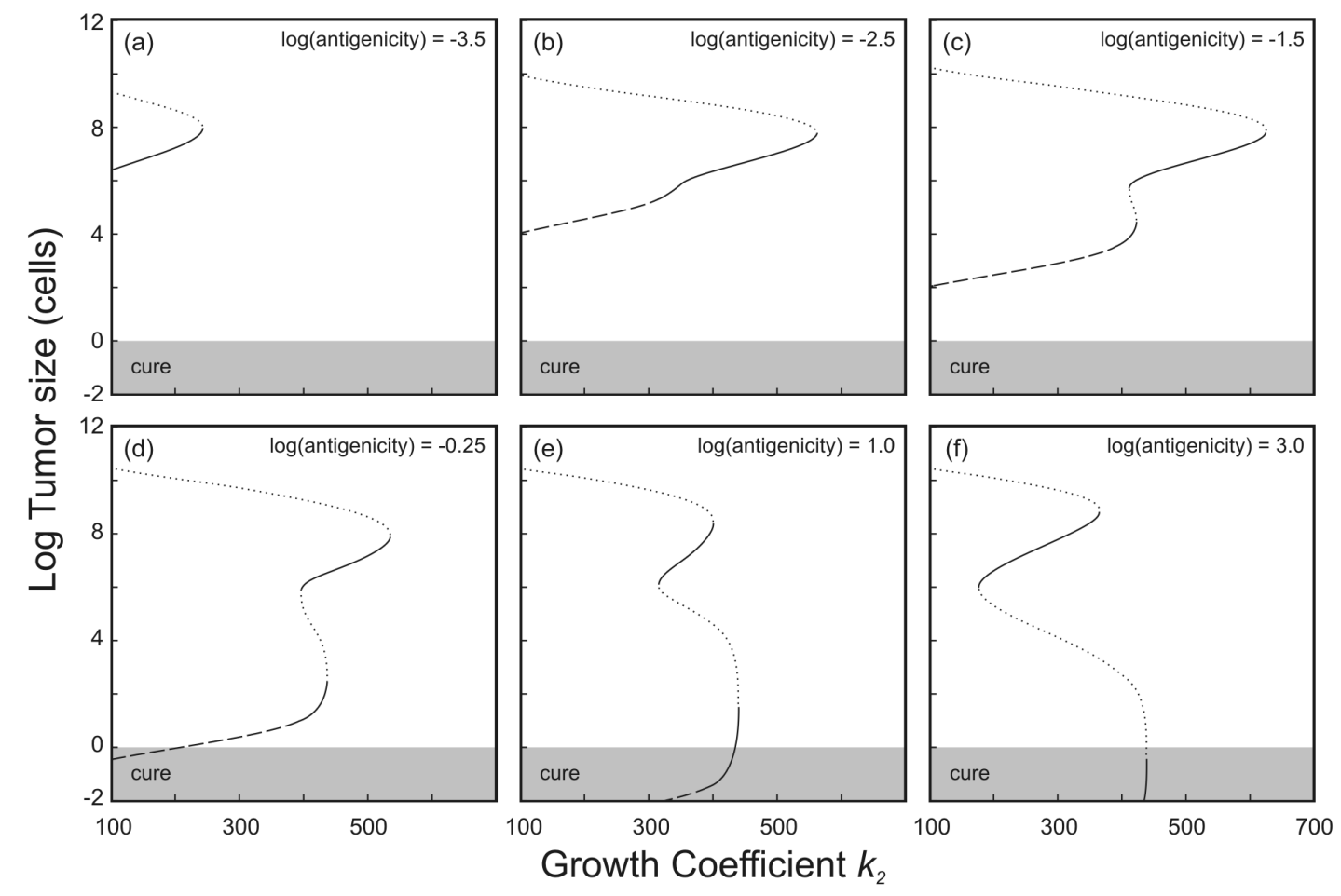

Figure 9: Bifurcation diagrams for various antigenicities. Solid lines indicate stable fixed points, dotted lines indicate unstable fixed points, and dashed lines indicate the unstable fixed points within a stable limit cycle. 


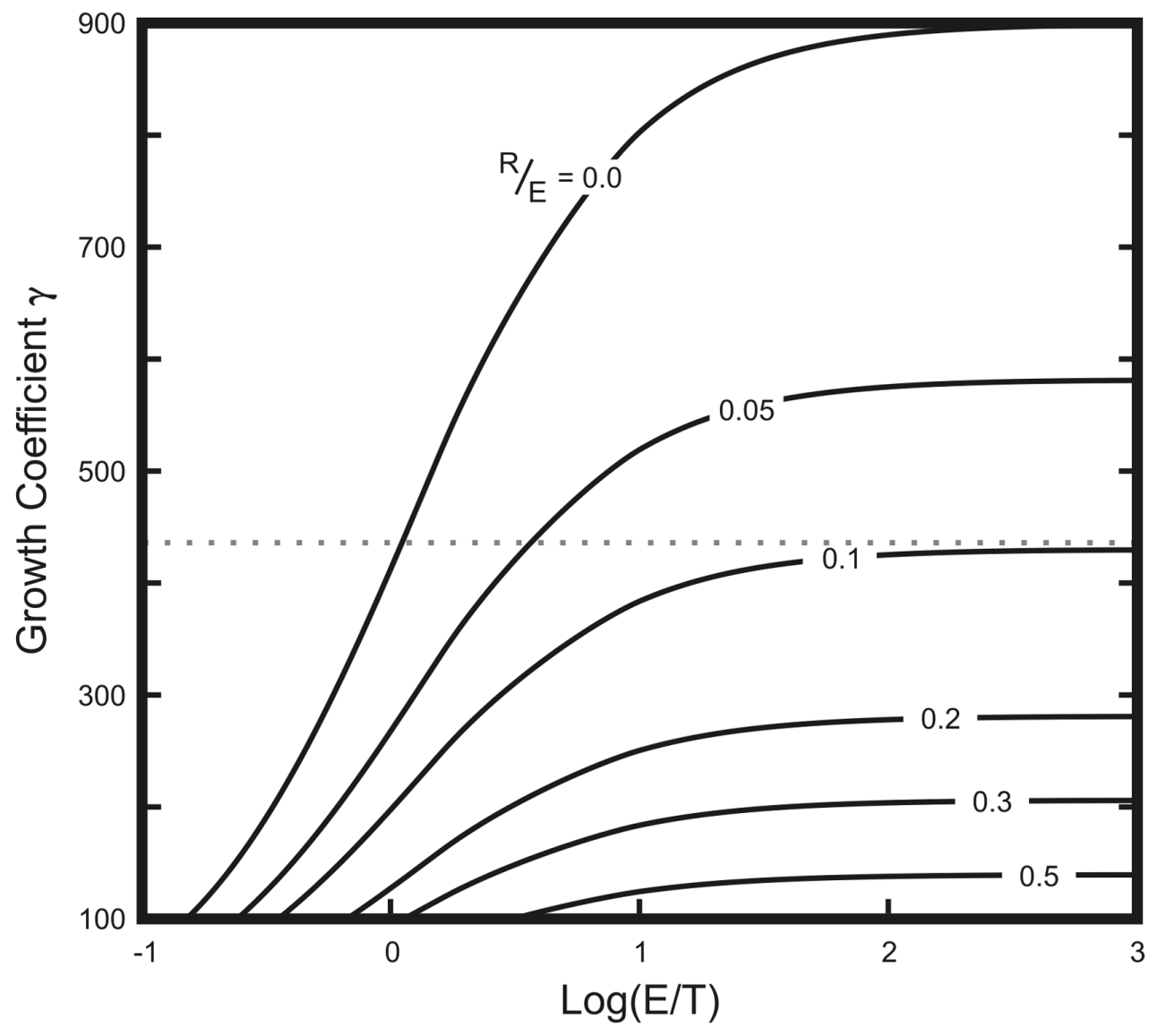

Figure 10: Solutions of Eq. (31) for fixed values of $R / E$. For a given $R / E$ ratio, combinations of $(\log (E / T), \gamma)$ that fall above the curve will lead to tumor growth, and combinations below the curve will lead to tumor removal. The curve for $R / E=0.1$ approaches the dotted asymptote shown at $\gamma=435$, which corresponds to the sharp upper edge of the transition from removal to growth in Figure 3. 
Table 1: Parameter values for tumor cells, Eq. (16)

\begin{tabular}{|c|c|c|c|}
\hline Parameter & Value & Units & Reference \\
\hline$\gamma_{1}$ & $100-1000$ & cell $^{1-\gamma} \cdot d_{a y}{ }^{-1}$ & $\begin{array}{l}\text { Friberg and Mattson (1997) } \\
\text { Iwashita et al. (1998) } \\
\text { Jackson et al. (1984) } \\
\text { Peer et al. (1993) }\end{array}$ \\
\hline$T_{1}$ & $10^{6}$ & cell & Steele (1977) \\
\hline$m$ & $\frac{1}{2}$ & & Hart et al. (1998) \\
\hline$n$ & $\begin{array}{l}\frac{2}{3} \\
3\end{array}$ & & Jain and Ward-Hartley (1984) \\
\hline $\begin{array}{l}p \\
r_{0}\end{array}$ & $\begin{array}{l}3 \\
0.9\end{array}$ & $d a y^{-1}$ & Dudley et al. (2002) \\
\hline$k_{1}$ & 100 & cell $l^{1-\nu}$ & $\begin{array}{l}\text { Jain and Ward-Hartley (1984) } \\
\text { Steele (1977) }\end{array}$ \\
\hline$k_{2}$ & 1.2 & & Dudley et al. (2002) \\
\hline$k_{3}$ & 11 & & Piccirillo and Shevach (2001) \\
\hline$S_{1}$ & 3.5 & $n g \cdot m l^{-1}$ & Thomas and Massagué (2005) \\
\hline
\end{tabular}

Table 2: Parameter values for dendritic cells, Eqs. (17) and (18)

\begin{tabular}{llll}
\hline Parameter & Value & Units & Reference \\
\hline$a$ & $10^{-5}-10^{5}$ & $d a y^{-1}$ & \\
$I_{1}$ & 0.4 & $n g \cdot m l^{-1}$ & Larmonier et al. (2007) \\
$R_{1}$ & $2 \times 10^{7}$ & cell & Larmonier et al. (2007) \\
$\lambda$ & 0.5 & $d a y^{-1}$ & Larmonier et al. (2007) \\
$\delta_{U}$ & 0.14 & $d a y^{-1}$ & Liu et al. (2007a) \\
$\delta_{D}$ & 0.5 & $d a y^{-1}$ & Liu et al. (2007a) \\
\hline
\end{tabular}


Table 3: Parameter values for T cells, Eqs. (19)-(24)

\begin{tabular}{llll}
\hline Parameter & Value & Units & Reference \\
\hline$\alpha_{1}$ & 23 & $d a y^{-1}$ & Mempel et al. (2004) \\
$\alpha_{2}$ & 16 & $d a y^{-1}$ & Mempel et al. (2004) \\
$\alpha_{3}$ & 9.9 & $d a y^{-1}$ & Mempel et al. (2004) \\
$\alpha_{4}$ & 1.9 & $d a y^{-1}$ & Mempel et al. (2004) \\
$\alpha_{5}$ & 5.1 & $d a y^{-1}$ & Mempel et al. (2004) \\
$\alpha_{6}$ & 2.1 & $d a y^{-1}$ & Mempel et al. (2004) \\
$\alpha_{7}$ & 0.022 & $d a y^{-1}$ & Liu et al. (2007b) \\
$k_{4}$ & 0.33 & & Mempel et al. (2004) \\
$C_{1}$ & 0.3 & $n g \cdot m l^{-1}$ & Thomas and Massagué (2005) \\
$S_{2}$ & 2.9 & $n g \cdot m l^{-1}$ & Thomas and Massagué (2005) \\
& 1.7 & $n g \cdot m l^{-1}$ & Liu et al. (2007b) \\
$S_{3}$ & 0.2 & $d a y^{-1}$ & Mempel et al. (2004) \\
$\delta_{A}$ & 1.0 & $d a y^{-1}$ & Yates and Callard (2001) \\
$\delta_{E}$ & 0.1 & $d a y^{-1}$ & Yates and Callard (2001) \\
$\delta_{H}$ & 0.1 & $d a y^{-1}$ & Yates and Callard (2001) \\
$\delta_{R}$ & & & Vukmanovic-Stejic et al. (2006) \\
\hline
\end{tabular}

Table 4: Parameter values for cytokines, Eqs. (25)-(27)

\begin{tabular}{|c|c|c|c|}
\hline Parameter & Value & Units & Reference \\
\hline$p_{C}$ & $1.7 \times 10^{-5}$ & $n g \cdot m l^{-1} \cdot$ cell $^{-1} \cdot d a y^{-1}$ & McKarns and Schwartz (2005) \\
\hline$p_{1}$ & $1.8 \times 10^{-8}$ & $n g \cdot m l^{-1} \cdot d a y^{-1} \cdot c e l l^{-1}$ & Liyanage et al. (2002) \\
\hline$p_{2}$ & $1.1 \times 10^{-7}$ & $n g \cdot m l^{-1} \cdot d a y^{-1} \cdot c e l l^{-1}$ & Danforth Jr and Sgagias (1996) \\
\hline$p_{3}$ & $1.4 \times 10^{-8}$ & $n g \cdot m l^{-1} \cdot d a y^{-1} \cdot c e l l^{-1}$ & Liyanage et al. (2002) \\
\hline$p_{4}$ & $1.3 \times 10^{-10}$ & $n g \cdot m l^{-1} \cdot d a y^{-1} \cdot$ cell $^{-1}$ & Gastl et al. (1993) \\
\hline$I_{2}$ & 0.75 & $n g \cdot m l^{-1}$ & $\begin{array}{l}\text { Taga et al. (1993) } \\
\text { de Waal Malefyt et al. (1993) }\end{array}$ \\
\hline$S_{4}$ & 0.9 & $n g \cdot m l^{-1}$ & $\begin{array}{l}\text { Thomas and Massagué (2005) } \\
\text { McKarns and Schwartz (2005) }\end{array}$ \\
\hline$\tau_{C}$ & 0.08 & day & $\begin{array}{l}\text { Konrad et al. (1990) } \\
\text { Lotze et al. (1985) }\end{array}$ \\
\hline$\tau_{S}$ & 0.07 & day & Wakefield et al. (1990) \\
\hline$\tau_{I}$ & 0.05 & day & Le et al. (1997) \\
\hline
\end{tabular}





\section{RECENT REPORTS}

41/09 Homogenization for advection-diffusion in a perforated domain Haynes

Hoang

Norris

Zygalakis

42/09 Fast stochastic simulation of biochemical reaction systems by al-

Melykuti ternative formulations of the Chemical Langevin Equation

Burrage

Zygalakis

43/09 Pseudoreplication invalidates the results of many neuroscientific

Lazic studies

44/09 Cardiac cell modelling: Observations from the heart of the cardiac physiome project

45/09 A Hybrid Radial Basis Function - Pseudospectral Method for Thermal Convection in a 3-D Spherical Shell

Flyer

46/09 Refining self-propelled particle models for collective behaviour

Yates

Baker

Erban

Maini

47/09 Stochastic Partial Differential Equations as priors in ensemble methods for solving inverse problems

Potsepaev

Farmer

Aziz

48/09 DifFUZZY: A fuzzy spectral clustering algorithm for complex data

Cominetti et al. sets

01/10 Fluctuations and instability in sedimentation

Guazzelli

Hinch

02/10 Determining the equation of state of highly plasticised metals from

Hinch boundary velocimetry

03/10 Stability of bumps in piecewise smooth neural elds with nonlinear adaptation

Kilpatrick

Bressloff

04/10 Random intermittent search and the tug-of-war model of motordriven transport

Newby

Bressloff

05/10 Ergodic directional switching in mobile insect groups

Escudero et al.

06/10 Derivation of a dual porosity model for the uptake of nutrients by root hairs

Zygalakis

Roose

07/10 Frost heave in compressible soils

Majumdar

Peppin

Style

Sander 
08/10 A volume-preserving sharpening approach for the propagation of sharp phase boundaries in multiphase lattice Boltzmann simula-

Dellar tions

09/10 Anticavitation and differential growth in elastic shells

Moulton

Goriely

10/10 On the mechanical stability of growing arteries

Goriely

Vandiver

11/10 Nonlinear Correction to the Euler Buckling Formula for Compressible Cylinders

De Pascalis

Destrade

Goriely

12/10 Nonlinear Morphoelastic Plates I: Genesis of Residual Stress

McMahon

Goriely

Tabor

13/10 Nonlinear Morphoelastic Plates II: Exodus to Buckled States

McMahon

Goriely

Tabor

14/10 Analysis of Brownian dynamics simulations of reversible biomolecular reactions

Lipkova

Zygalakis

Chapman

Erban

15/10 Travelling waves in hyperbolic chemotaxis equations Xue

Hwang

Painter

Erban

16/10 The Physics and Mechanics of Biological Systems

Goriely

Moulton

17/10 Crust formation in drying colloidal suspensions

Style

Peppin

Copies of these, and any other OCCAM reports can be obtained from:

Oxford Centre for Collaborative Applied Mathematics

Mathematical Institute

24 - 29 St Giles'

Oxford

OX1 3LB

England

www.maths.ox.ac.uk/occam 